\title{
Die Bundesländer in der nationalen Energie- und Klimapolitik: Räumliche Verteilungswirkungen und föderale Politikgestaltung der Energiewende
}

\author{
Jochen Monstadt $^{1} \cdot$ Stefan Scheiner $^{2}$
}

Eingegangen: 12. Mai 2015 / Angenommen: 20. April 2016 / Online publiziert: 24. Mai 2016

(c) Springer-Verlag Berlin Heidelberg 2016

Zusammenfassung Studien zur deutschen Energiewende befassen sich bislang vor allem mit Vorgängen der nationalen und kommunalen Energie- und Klimapolitik. Allerdings bringen die Bundesländer raumspezifische Verteilungsinteressen in die Politikgestaltung ein, die im föderalen Verhandlungssystem zu verarbeiten sind. So resultieren aus den verschiedenen klimapolitischen Maßnahmen des Bundes mitunter erhebliche, jedoch räumlich höchst ungleich verteilte Kosten- und Nutzeneffekte für die Energiewirtschaft, die Energie verbrauchenden Sektoren und öffentlichen Haushalte. Entsprechend variieren auch die regionalwirtschaftlichen und (partei-)politischen Interessen der Bundesländer, die diese mit ihren eigenständigen Klimaschutzprogrammen und Positionen in der föderalen Politikgestaltung verfolgen. Aufbauend auf einer Analyse der regionalwirtschaftlichen Interessen und klimapolitischen Programmatiken der Bundesländer zeigt der Artikel anhand der Förderung erneuerbarer Energien und des Emissionshandels, wie föderale Verteilungskonflikte bewältigt wurden und klimapolitische Handlungsfähigkeit hergestellt wurde.

Prof. Dr. Jochen Monstadt

j.monstadt@iwar.tu-darmstadt.de

Dipl.-Umweltwiss. Stefan Scheiner

s.scheiner@iwar.tu-darmstadt.de

1 Fachgebiet Raum- und Infrastrukturplanung, Fachbereich Bau- und Umweltingenieurwissenschaften, Technische Universität Darmstadt,

Franziska-Braun-Str. 7, 64287 Darmstadt, Deutschland

2 Fachgebiet Raum- und Infrastrukturplanung, Fachbereich Bau- und Umweltingenieurwissenschaften, Technische Universität Darmstadt, Franziska-Braun-Str. 7, 64287 Darmstadt, Deutschland
In den Handlungsfeldern wurden redistributive Politiken über Konzessionen an einzelne Bundesländer und distributive Mechanismen durchgesetzt; Kostenexternalisierungsstrategien kommt eine besondere Bedeutung $\mathrm{zu}$, und die EU konnte teilweise Blockaden in der föderalen Politikgestaltung auflösen. Entgegen den verbreiteten Annahmen der deutschen Föderalismusforschung haben klimapolitische Entscheidungen den regionalen Wettbewerb partiell erhöht, was sich teilweise innovationsfördernd auswirkt. Allerdings verdeutlicht die Analyse der Handlungsfelder auch, dass die Durchsetzung regionalwirtschaftlicher Interessenlagen die Kosteneffizienz und die Effektivität der deutschen Energiewendepolitik teilweise gemindert hat.

Schlüsselwörter Energiewende · Föderalismus · Räumliche Verteilungswirkungen von Umweltpolitiken · Energie- und Klimapolitik

\section{The Länder in the German energy and climate policy: Distributional issues and federal decision- making}

Abstract Until today, the academic and political debates on the German energy transition have primarily focused on policy activities and processes at either the national or local level. However, they have largely ignored how the Länder shape the federal decision-making on the allocation of greenhouse gas emissions according to their place-based economic interests. The starting point of this paper is the empirical evidence that effective national climate mitigation policies can go along with considerable and spatially unevenly distributed redistributive effects for regional energy industries, energy consumption sectors and thus public budgets of the Länder. Since the costs and benefits of cli- 
mate mitigation policies are distributed unevenly, the Länder pursue place-based economic and political interests that shape their climate mitigation programs and their positions in federal decision-making. Based on an analysis of the regional economic interests and of the climate mitigation programs of the Länder, the paper uses the cases of the renewable energy policies and the European emission trading system to demonstrate how (re-)distributional conflicts in climate mitigation policies have been reconciled. The case studies show that redistributive policies could be implemented through concessions to single Länder. They also show that through distributive mechanisms, the externalization of costs to the users played a major role, and that the EU could partially dissolve blockades in decision-making. Contrary to findings in the academic debate on German federalism, federal climate mitigation policies have partially stimulated mechanisms for economic and innovation competition between the Länder. However, the findings also demonstrate that the concessions to regional economic interests have reduced the cost-efficiency and effectiveness of the German energy and climate mitigation policies.

Keywords Energy transition - Federalism - Spatial distribution effects of environmental policies - Energy and climate policy

\section{Einleitung}

Analysen zur deutschen Energiewende befassen sich bislang überwiegend mit der Bundespolitik, die in Interaktion mit der internationalen und europäischen Politik als wichtigste staatliche Ebene aufgefasst wird. Zugleich sind die kommunale und regionale Ebene in den letzten Jahren vermehrt in das Blickfeld der Energie- und Raumforschung gerückt (vgl. Kern 2008; Bulkeley/Broto/Edwards 2012). Wenig berücksichtigt wird hierbei, dass sich die deutsche Energiewende innerhalb einer föderalen Staatsorganisation vollzieht, in welcher die Bundesländer die Politikgestaltung wesentlich beeinflussen. Unklar ist bisher geblieben, inwieweit die wirtschaftlichen und politischen Interessenlagen und Konflikte der Bundesländer sowie die institutionellen Rahmenbedingungen und Dynamiken des deutschen Föderalismus die Energiewende vorantreiben oder hemmen.

Dass Klimapolitik in der Stromversorgung ein Handlungsbereich ist, der für subnationale Akteure gerade in föderalen Systemen ein wichtiges Politikfeld darstellt, zeigt sich in zahlreichen Studien zur Klimapolitik anderer Föderalstaaten, etwa zu den USA (vgl. unter anderem Peterson/Rose 2006; Rabe 2009; Schlager/Engel/Rider 2011), zu Australien (Crowley 2007; Bailey/Maresh 2008), Spanien (Tàbara 2007; Hanf/Morata 2012) und Kanada (Harrison 2007; Macdonald 2008). Insgesamt zeigen diese, dass Kli- maschutz eine politische Herausforderung ist, bei der die einzelnen subnationalen Akteure in Abhängigkeit von ihrer regionalen Wirtschaftsstruktur und geographischen Bedingungen sehr unterschiedliche Interessen verfolgen. Diese subnationalen Interessen stehen dabei immer in einer engen Beziehung zu den jeweiligen föderalen Institutionen und den damit verbundenen Logiken der Politikgestaltung. Die stark dezentral ausgerichtete föderale umweltpolitische Kompetenzverteilung in Australien, Spanien und teilweise auch den USA haben es beispielsweise möglich gemacht, dass die subnationalen Akteure maßgebliche klimapolitische Initiativen vorantreiben konnten (Tàbara 2007; Schlager/Engel/Rider 2011), während die faktisch schwache Position des Zentralstaates im föderalen System Kanadas der Provinz Alberta umfangreiche Vetomöglichkeiten in der Klimapolitik eröffnet (Macdonald 2008).

Die Energiewende wird in Deutschland seit den 1980erJahren politisch diskutiert und umgesetzt (vgl. Monstadt 2004). Durch den Ausstieg aus der Atomenergie und die Einführung effektiver Klimaschutzmaßnahmen leitet diese in der Stromversorgung einen tiefgreifenden Transformationsprozess ein. In den föderalen Entscheidungsprozessen kommen jedoch sehr unterschiedliche regionale Wirtschaftsstrukturen und sehr heterogene subnationale Interessenlagen zum Tragen. Zum einen unterscheiden sich die Potenziale zur Nutzung regenerativer Energien zwischen den Bundesländern aufgrund naturräumlicher, siedlungsund wirtschaftsstruktureller Gegebenheiten erheblich. Zum anderen konzentrieren sich die energieintensiven Industrien und klassischen Stromerzeugungskapazitäten auf einzelne Regionen (vgl. Zimmermann 2005). Unweigerlich führt eine Transformation der Stromversorgung dabei zu einer räumlich ungleichen Verteilung der Kosten für die Energiewirtschaft und energieintensive Industrien oder auch der ökonomischen Nutzen der erneuerbaren Energiewirtschaft. Mit der Gestaltung der Energiewende gehen damit manifeste regionale Wirtschaftsinteressen und komplexe interregionale Verteilungskonflikte einher. Die Ausgangsthese des folgenden Artikels ist daher, dass im Zuge der Politiken zur Energiewende nicht nur über die Verteilung von Emissionsrechten zwischen einzelnen Sektoren (z. B. Energiewirtschaft, Verkehr, Bauen, Land- und Forstwirtschaft), sondern auch über Standortinteressen und die räumliche Verteilung von Kosten und ökonomischen Nutzen verhandelt wird.

Dass die deutsche Klimapolitik trotz regionaler (Um-) Verteilungseffekte im internationalen Vergleich als verhältnismäßig erfolgreich beurteilt wird, überrascht dabei vor allem auch im Lichte der politikwissenschaftlichen Föderalismusdebatte. Diese schreibt dem konsensorientierten föderalen Verhandlungssystem in Deutschland für Handlungsbereiche mit starken regionalen Verteilungseffekten eher ein geringes Potenzial zur Problemlösung zu (vgl. 
Scharpf 2009). Die hierdurch provozierten Risiken von Entscheidungsblockaden können sich noch verschärfen, wenn sich diese mit einem ausgeprägten Parteienwettbewerb verschränken (Lehmbruch 2000). Dies ist gerade im Bereich der Energie- und Klimapolitik der Fall, die traditionell als ein parteipolitisch stark polarisiertes Handlungsfeld gilt. Parteipolitisch kontroverse Energie- und Klimapolitiken mit regionalen (Um-)Verteilungseffekten bergen daher erhebliche Risiken politischer Blockadesituationen, die effektive und kosteneffiziente Sachentscheidungen unwahrscheinlich machen. Das bundesdeutsche föderale System so der Befund - sieht eine Vielzahl von Veto-Möglichkeiten vor, mittels derer die Bundesländer auf Bundesebene intervenieren bzw. sich einer Umsetzung nationaler Ziele entziehen können.

Im Mittelpunkt dieses Artikels stehen daher folgende Fragen: Welches sind die energiewirtschaftlichen Standortinteressen der Bundesländer und inwiefern schlagen sich diese in den subnationalen Klimaprogrammatiken nieder? Wie prägen die räumlichen Verteilungswirkungen der Klimaschutzpolitiken das interessengeleitete energiepolitische Handeln der Bundesländer in der föderalen Politikgestaltung? Wie kann die Entscheidungsfähigkeit der deutschen Klimapolitik angesichts der heterogenen energiewirtschaftlichen und -politischen Interessen der Bundesländer erklärt werden und welchen Einfluss haben diese auf die Entscheidungsergebnisse? Ziel ist es also, die räumlichen Verteilungswirkungen und regionalwirtschaftlichen Interessenlagen der Bundesländer zu identifizieren, um vor dem Hintergrund der föderalen Politikgestaltung eine kritische Bilanz zur Effektivität und Effizienz der deutschen Energie- und Klimapolitik zu ziehen. Dies geschieht, indem zunächst föderalismustheoretische Debatten um die institutionellen Bedingungen des kooperativen Föderalismus zusammengefasst und untersuchungsleitende Hypothesen formuliert werden, unter welchen Voraussetzungen verteilungsrelevante Entscheidungen möglich sind (Abschn. 2). AnschlieBend werden die unterschiedlichen und zwischen Bund und Ländern wenig abgestimmten klimapolitischen Programmatiken der Bundesländer untersucht, um die subnationalen Standortinteressen herauszuarbeiten (Abschn. 3). Dem folgt eine Analyse der beiden wichtigsten energiebezogenen Handlungsfelder der deutschen Klimapolitik - der Förderung erneuerbarer Energien und des Emissionshandels. ${ }^{1}$ An diesen Handlungsfeldern wird demonstriert, dass die räumlich ungleiche Verteilung der Kosten und Nutzen einer Reduktion von Treibhausgasmissionen das interessen-

\footnotetext{
${ }_{1}^{1}$ Die Erneuerbare-Energien-Politik in Deutschland weist eine im internationalen Vergleich erfolgreiche Bilanz auf und hat dazu beigetragen, dass der Anteil der erneuerbaren Energien in der Stromerzeugung in den Jahren 1990 bis 2014 von 3 auf ca. $27 \%$ gesteigert werden konnte (BDEW 2014). Der $\mathrm{CO}_{2}$-Emissionshandel, der in der gesamten EU gilt, reguliert etwa die Hälfte der deutschen $\mathrm{CO}_{2}$-Emissionen.
}

geleitete Handeln der Bundesländer in der föderalen Klimapolitik wesentlich prägt (Abschn. 4). In den Schlussfolgerungen werden der Nutzen der föderalismustheoretischen Diskussion für die Erklärung der Dynamiken und Ergebnisse der deutschen Energiewendepolitiken kritisch reflektiert. Zugleich wird eine kritische Bilanz zur Effektivität und Effizienz der Energie- und Klimapolitik gezogen und es werden Handlungsbedarfe aufgezeigt (Abschn. 5).

Der Artikel basiert auf Ergebnissen eines international vergleichenden Forschungsprojektes zur Allokation von Treibhausgasemissionen in Föderalstaaten. ${ }^{2}$ Empirische Grundlage bildet die Auswertung eines breiten Spektrums von Dokumenten (unter anderem Klimaprogramme, Parlamentsprotokolle, Zeitungsartikel), statistischen Daten und von 16 leitfadengestützten Interviews mit Experten der Energie- und Klimapolitik, die zwischen 2010 und 2014 durchgeführt wurden. ${ }^{3}$

\section{Konfliktkonstellationen und Verhandlungslogiken im kooperativen Föderalismus}

Die Gestaltung der Energiewendepolitik in Deutschland erfolgt innerhalb eines föderalen Verhandlungssystems zwischen Bund und Ländern, das durch eine Vielzahl wechselseitiger Abhängigkeiten geprägt ist. Zwar liegen wesentliche Gesetzgebungskompetenzen in der deutschen Energie- und Klimaschutzpolitik beim Bund (Weidner/Eberlein 2009: 334; Rodi/Sina 2011), allerdings nehmen die Bundesländer auf verschiedene Weise Einfluss.

Erstens sind die Bundesländer an der Gesetzgebung des Bundes zu beteiligen, sofern diese - wie unter anderem bei der Einführung des Emissionshandels - ihre fiskalischen und administrativen Angelegenheiten direkt berührt (Hrbek 2004). Ihnen ist es bei solchen zustimmungspflichtigen Gesetzen möglich, über den Bundesrat ihr Veto einzulegen.

\footnotetext{
${ }^{2}$ Hierbei handelt es sich um das vom Canadian Social Science and Humanities Research Council geförderte Projekt „Allocating Canadian greenhouse gas emission reductions amongst sources and provinces: learning from Germany and the EU“" (Fördernr. 865-2008-0073).

${ }^{3}$ Hierbei wurden spezifische Wissens- und Deutungsmuster von Experten eingeholt und verglichen, die zusammen ein möglichst breites Spektrum von Entscheidungsträgern abdecken. Gegenstand der Interviews war vor allem die Frage, wie die räumlichen Verteilungswirkungen der beiden untersuchten Handlungsfelder die föderale Politikgestaltung der Energiewende beeinflusst haben, und wie Handlungsblockaden vermieden werden konnten. Befragt wurden Vertreter aus Umwelt- und Wirtschaftsministerien des Bundes und der Bundesländer, des Umweltbundesamtes, der Emissionshandelsstelle, des Umweltrates, der Verbände der traditionellen und regenerativen Energiewirtschaft, aus Umwelt- und Verbraucherschutzverbänden sowie von Energieunternehmen. Die Auswertung der qualitativen Leitfadeninterviews orientiert sich an den von Meuser und Nagel (2005: $80 \mathrm{ff}$.) skizzierten Methoden.
} 
Darüber hinaus sind Kooperationsgremien seit Gründung der Bundesrepublik Deutschland ein wesentlicher Bestandteil der Koordination zwischen den Regierungen von Bund und Ländern (Kropp 2010: 127). Die freiwillige Zusammenarbeit umfasst eine Fülle kooperativer Arrangements, etwa Ausschüsse, Arbeitsgemeinschaften, Kommissionen, Arbeitskreise und (Fach-)Ministerkonferenzen. Diese können sowohl eine vertikale (Bund-Länder) als auch horizontale (zwischen Bundesländern) Koordination bzw. sowohl bi- als auch multilaterale Aushandlungen umfassen (vgl. Zimmer 2010: 677; Hegele/Behnke 2013).

Zweitens haben die Bundesländer die Möglichkeit, eigenständige, von Bundesregelungen abweichende rechtliche Regelungen der Naturschutz- und Raumordnungspolitik zu treffen (z. B. Landesplanungsgesetze, Windkrafterlasse) - beides wichtige Handlungsfelder für die Förderung bzw. Behinderung des Ausbaus erneuerbarer Energien (vgl. Ehlers/Böhme 2011; Diller 2015). So unterscheiden sich beispielsweise die in Windenergieerlassen der Länder definierten Vorgaben für die Planung und Genehmigung von Windenergieanlagen. Zugleich wurden in den meisten Bundesländern Eignungsgebiete für die Windenergie bei gleichzeitiger Ausschlusswirkung an anderen Standorten ausgewiesen, während einzelne Länder (Saarland, Baden-Württemberg, Bayern, Hessen) den Kommunen zuletzt deutlich größere Freiheiten bei der Festlegung von Windeignungsstandorten eingeräumt haben (Diller 2015).

Drittens zeigt die Diffusionsforschung, dass die Bundesländer auch außerhalb rechtlicher Vorgaben als innovative Vorreiter der Energie- und Klimapolitik agieren können. Teilweise auf Initiative der Länder, teilweise auch in „Energieregionen“ unterschiedlicher räumlicher und institutioneller Konfiguration (Monstadt 2007) oder durch Förderung lokaler Initiativen (Kern 2008) implementieren und erproben die Bundesländer neue politische Instrumente, wie Klimaschutzprogramme, Energiekonzepte, Wirtschaftskooperationen, Selbstverpflichtungen (vgl. Bruns/ Ohlhorst/Wenzel et al. 2011: 375; Jörgensen 2012). Zugleich wird die regionale Wirtschaftsförderung wesentlich von den Ländern gestaltet. Sowohl die direkte Unterstuitzung von Unternehmen bzw. Unternehmensansiedlungen und wirtschaftsnahen Infrastrukturen (z. B. Kompetenzzentren, Energieagenturen, Innovationsnetzwerken) als auch die Finanzierung der innovationsrelevanten Bereiche Bildung und Forschung wird mehr als zur Hälfte von den Bundesländern getragen (Hellmann/Kullas 2012).

Neben einer institutionellen Struktur, innerhalb derer die Aufgabenwahrnehmung der verschiedenen politischen Ebenen stark miteinander verflochten ist, führt die Struktur des parteipolitischen Systems zu hohen Kooperationszwängen (Lehmbruch 2000; Kropp 2010). Da die Parteien sowohl auf Landes- als auch auf Bundesebene agieren, spielen im Bundesrat neben regionalen Interessen auch parteipo- litische Interessen eine große Rolle. Besondere Dynamiken und Herausforderungen entstehen insbesondere dann, wenn bei zustimmungspflichtigen Vorhaben die jeweiligen Mehrheiten in Bundestag und Bundesrat nicht konvergieren (Lehmbruch 2000; Haas/Jun/Niedermayer 2008) - so z. B. bei der früheren rot-grünen Bundesregierung ab dem Jahr 1999 und der konservativ-liberalen Regierungskoalition ab Mai 2011. Zwar besteht über das allgemeine Ziel „Klimaschutz" ein parteipolitischer Konsens, jedoch zeigen sich klare Unterschiede zwischen den traditionellen politischen „Blöcken“ SPD/Grüne und CDU/CSU/FDP bei der Frage, wie der energiebezogene Klimaschutz gestaltet werden soll (Mez 2003).

Die Gestaltung der Energiewende findet daher in einem Spannungsfeld statt, das in der Föderalismusforschung als große Herausforderung für die politische Gestaltungsfähigkeit bewertet wird. Die Verschränkungen von Föderalismus und Parteienwettbewerb resultieren in einem oftmals sehr hohen Abstimmungsaufwand, bei dem die politischen Akteure gezwungen sind, einen möglichst breiten Konsens zu suchen (Scheller 2008: 24; Kropp 2010). Insbesondere bei Handlungsfeldern, die starke räumliche (Um-) Verteilungseffekte mit sich bringen, oder die parteipolitisch stark polarisieren, ergeben sich ,erhebliche Schranken der politischen Handlungsfähigkeit" (Scharpf 1976: 21). Diese machen Blockadesituationen bei Sachentscheidungen und Immobilismus in den Bund-Länder-Abstimmungen wahrscheinlich (Scharpf 1976; Lehmbruch 2000). Die empirische Förderalismusforschung verweist darauf, dass Strategien der Besitzstandswahrung bzw. Entscheidungsblockaden häufig dadurch aufgelöst werden, dass entweder distributive Politiken favorisiert oder Strategien zur Abwälzung der Kosten auf Akteure außerhalb des föderalen Verhandlungsrahmens gewählt werden (WachendorferSchmidt 2005: 100). Entsprechend den Befunden der Föderalismusforschung kann daher auch in den Bund-LänderVerhandlungen zur Gestaltung der Energiewende erwartet werden, dass diese von Strategien der Besitzstandswahrung begleitet werden oder dass die Kosten energiepolitischer Reformen auf Dritte externalisiert werden.

Neben dieser Externalisierung der Kosten können Immobilismen aber auch durch Anstöße von Akteuren außerhalb des föderalen Verhandlungssystems gelöst werden. Von Bedeutung sind in diesem Zusammenhang unabhängige Institutionen wie das Bundesverfassungsgericht oder auch die Europäische Union (EU). Viele Politikfelder, insbesondere aber die Klimapolitik (Wurzel 2010), sind verstärkt durch Mehrebenenverflechtung mit eigenen Dynamiken politischer Gestaltung geprägt (Benz 1998). Der EU wird dabei zugeschrieben, eine eher entscheidungsfördernde Wirkung auf föderale Verhandlungen zu haben und Blockaderisiken zu mindern (Lehmbruch 2000; WachendorferSchmidt 2005). Insofern ist auch bei der Gestaltung der 


\section{3}

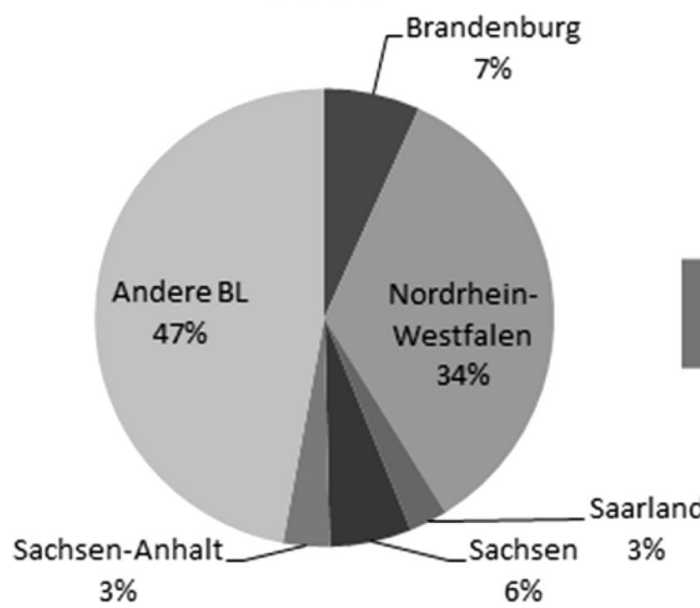

2011

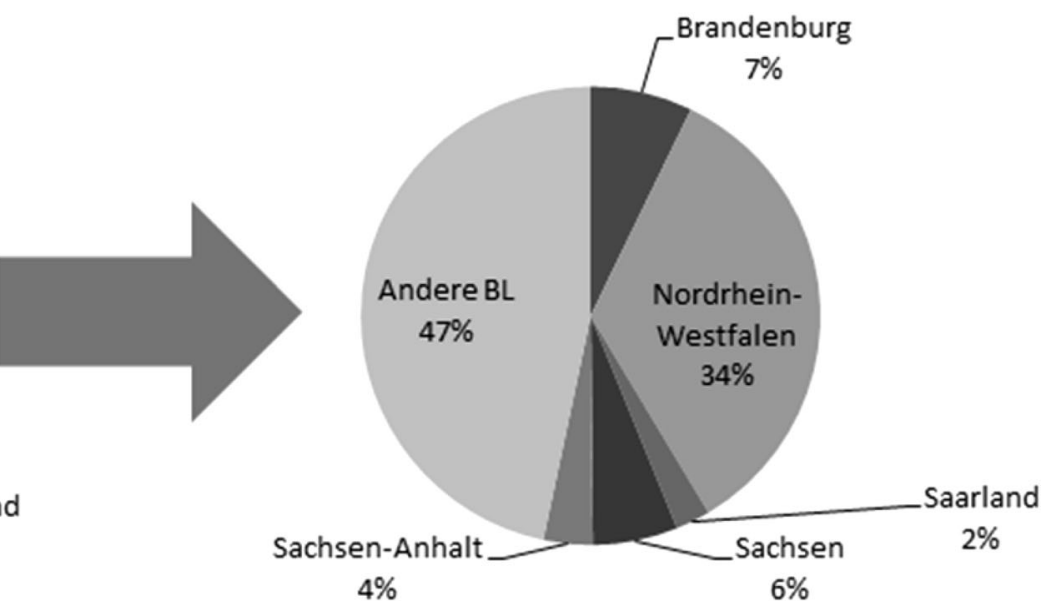

Abb. 1 Anteile der karbonintensiven Bundesländer (BL) an den energiebedingten $\mathrm{CO}_{2}$-Emissionen (Jahre 2003 und 2011). (LAK Energiebilanzen 2016)

Energiewende zu erwarten, dass sich progressive Energieund Klimapolitiken der EU entscheidungsfördernd auf die föderalen Verhandlungen um die deutsche Energiewende auswirken.

Infolge der kooperativen Ausrichtung des deutschen föderalen Systems und des verfassungsrechtlichen Anspruchs auf gleichwertige Lebensverhältnisse wird in der Forschung zum deutschen Föderalismus davon ausgegangen, dass eigenständige subnationale Politiken - ausgelöst etwa durch föderalen Wettbewerb oder lernorientierte Diffusionsprozesse - eine deutlich geringere Rolle spielen als in wettbewerbsföderalen Systemen der USA oder der Schweiz (Blancke 2004: 21-23; Benz 2007b). Als Ausdruck dieser Unitarisierung wird der umfangreiche Katalog an Bereichen aufgeführt, in denen die Bundesebene die Gesetzgebungskompetenz besitzt, und auf den gemeinsamen Steuerverbund und den Länderfinanzausgleich verwiesen (Scharpf 2009: 21-26). Zwar wird an den oben skizzierten Beispielen deutlich, dass einzelne Bundesländer eine energie- und klimapolitische Vorreiterfunktion übernehmen können. Die Einschätzung ist somit insofern zu relativieren, als Bundesländer (wie auch Kommunen) insbesondere jenseits von Rechtssetzung als wichtige innovative „Laboratorien“ zur Weiterentwicklung des Politikfeldes beitragen können. Es ist aber dennoch zu erwarten, dass die faktische Unitarisierung von Gesetzgebung einen politischen Wettbewerb zwischen den Bundesländern stark einschränkt (vgl. Braun 2004: 144-145; Benz 2007a) und eine solche Dynamik anders als etwa in wettbewerbsföderalen Staaten (vgl. Rabe 2009) bei der Gestaltung der Energiewende daher keine wichtige Rolle spielt.

\section{Föderale Konfliktkonstellationen in der deutschen Klimapolitik vor dem Hintergrund wirtschaftlicher Standortinteressen}

Die Umsetzung von nationalen Reduktionszielen von Treibhausgasemissionen geht mit erheblichen räumlichen Umverteilungseffekten einher, sowohl was die Kosten für die Energiewirtschaft und energieintensive Industrien als auch den ökonomischen Nutzen durch den Ausbau der erneuerbaren Energiewirtschaft betrifft (vgl. Monstadt/Scheiner 2014). Unter einem besonderen ökonomischen Anpassungsdruck stehen dabei vor allem die traditionellen „Energie-Bundesländer“, die sich durch eine stark ausgeprägte Kohlewirtschaft und zum Teil auch eine hohe Konzentration energieintensiver Betriebe auszeichnen, was sich in überdurchschnittlich hohen $\mathrm{CO}_{2}$-Emissionen im Verhältnis zur Einwohnerzahl und Wirtschaftsleistung niederschlägt. Als solche „karbonintensiven“ Bundesländer können vor allem Nordrhein-Westfalen, Brandenburg, Sachsen, Sachsen-Anhalt und das Saarland bezeichnet werden. So entfällt etwa ein Drittel der nationalen $\mathrm{CO}_{2}$-Emissionen auf Nordrhein-Westfalen und auch die anderen karbonintensiven Bundesländer weisen trotz ihrer relativ geringen Bevölkerungszahl hohe Anteile an den $\mathrm{CO}_{2}$-Emissionen auf. Ihre regionalen Anteile haben sich seit 2003 kaum verschoben (siehe Abb. 1). Da Ende 2014 deutlich wurde, dass die nationalen $\mathrm{CO}_{2}$-Emissionen zukünftig deutlich stärker als bisher gesenkt werden müssen, um das deutsche Klimaziel bis 2020 erreichen zu können (vgl. BMUB 2014), fallen insbesondere in den karbonintensiven Bundesländern überproportional hohe Kosten einer Modernisierung der Stromerzeugung und -nachfrage an. 


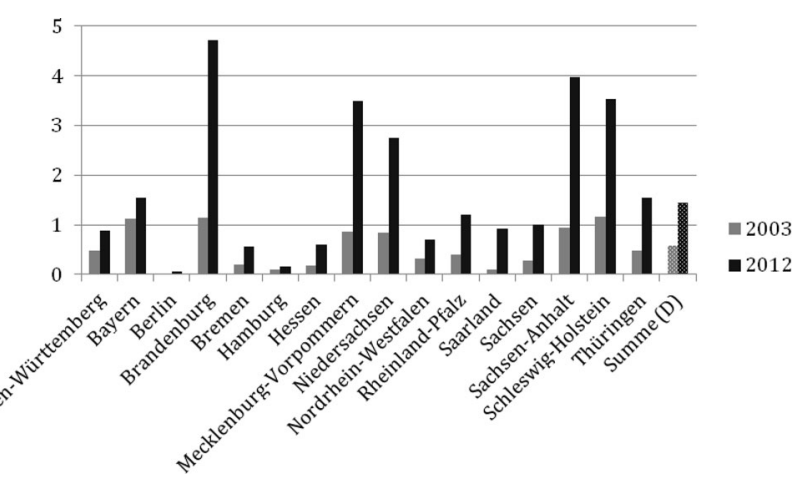

Abb. 2 Stromproduktion durch die Nutzung erneuerbarer Energien pro Einwohner in den Bundesländern (GWh/1000 Einw.) für 2003 und 2012. (LAK Energiebilanzen 2016; AGEE-Stat 2015)

Dagegen zeigt sich, dass die ökonomische Bedeutung der klassischen Energieländer beim Ausbau der erneuerbaren Energien innerhalb des letzten Jahrzehnts zum Teil deutlich geringer ist (siehe Abb. 2). Zwar ist etwa Nordrhein-Westfalen auch bei den erneuerbaren Energien einer der wichtigsten Standorte, allerdings zeigt die Entwicklung von 2003 bis 2012, dass andere Länder den Ausbau deutlich dynamischer vorantreiben.

Wie durch die Analyse der subnationalen Klimaprogrammatiken seit dem Jahr 2000 aufgezeigt werden kann, sind sich die Bundesländer dieser räumlichen Umverteilungsdimension deutlich bewusst und die energiewirtschaftlich unterschiedlichen Bedingungen stellen eine wesentliche Bestimmungsgröße für die föderalen Konfliktkonstellationen in der Klimapolitik dar. Am deutlichsten wird dies anhand der Klimaschutzprogramme der kohlewirtschaftlich stark geprägten Bundesländer Nordrhein-Westfalen und Saarland, deren $\mathrm{CO}_{2}$-Minderungsziele vergleichsweise wenig ambitioniert sind. Zwar identifiziert das Saarland im Klimaschutzgutachten von 1998 das „saarländische“ Potenzial einer Minderung der $\mathrm{CO}_{2}$-Emissionen um $24 \%$ bis 2005; allerdings wird hervorgehoben, dass dieses Potenzial vor allem mittels bundespolitischer Maßnahmen „mobilisiert“ werden müsse (vgl. Landesregierung Saarland 1998). Im Klimaschutzkonzept aus dem Jahre 2008 verzichtet das Saarland dann unter Berufung auf die eingeschränkten landespolitischen Spielräume sogar auf ein eigenes $\mathrm{CO}_{2}$-Minderungsziel. Nordrhein-Westfalen hat mit seinen Klimaschutzkonzepten von 2001 und 2005 umfangreiche Maßnahmenkataloge verabschiedet, allerdings wird bei den formulierten Landeszielen im Jahr 2001 nur eine Minderung um $11 \%$ bis 2005 als möglich erachtet, die landespolitisch in erster Linie durch die Erneuerung der (Kohle-)Kraftwerke erreicht werden soll. Diese Überzeugung, $\mathrm{CO}_{2}$-Minderungen vor allem durch Modernisierungen der Kohlekraftwerke und nachfrageseitige Effizienzmaßnahmen erreichen zu können, ist in Nordrhein-Westfalen auch in der folgenden Energie- und Klimaschutzstrategie aus dem Jahr 2008 und im landeseigenen Klimaschutzgesetz erkennbar. ${ }^{4}$ Die skizzierte Zurückhaltung in beiden Ländern erklärt sich durch die starke regionale Bedeutung der Kohlewirtschaft, aber auch der energieintensiven Industrien. So zeichnen sich beide Bundesländer (neben Brandenburg) durch den höchsten Anteil von durch "Carbon Leakage“ gefährdeten Arbeitsplätzen aus - einer Verlagerung von Industriestandorten aufgrund höherer Produktionskosten durch Klimaschutzmaßnahmen (Jakubowski/Zarth 2009: 7). Dagegen sehen die Landesregierungen in NordrheinWestfalen und im Saarland bis heute deutlich geringere Möglichkeiten, die etablierte Stromproduktion durch erneuerbare Energien zu ersetzen. Ihre Zielsetzungen in diesem Bereich liegen mit $20 \%$ bis 2020 (Saarland) bzw. $30 \%$ bis 2025 (Nordrhein-Westfalen) klar unter dem Bundesziel von $35 \%$ bis 2020 (vgl. Breyer/Müller 2013).

Ähnliche Effekte insbesondere aufgrund der großen strukturellen Bedeutung der Kohlewirtschaft zeigen sich auch in den Energie- und Klimaprogrammen der karbonintensiven Bundesländer Brandenburg, Sachsen und SachsenAnhalt. Während ihre Klimaziele lange kaum über dem Niveau der in den 1990er-Jahren erreichten Minderungen lagen (vgl. Schleich/Eichhammer/Böde et al. 2001), formuliert Brandenburg erst in seinem Klimaschutzprogramm 2008 und seiner Energiestrategie 2012 ambitionierte $\mathrm{CO}_{2}$ Reduktionsziele. Sachsen-Anhalt visiert zwar weitergehende Reduktionen nicht explizit an, hält diese aber aufgrund der energiewirtschaftlichen Entwicklungen im Bundesland für wahrscheinlich. Auch wenn beide Bundesländer ebenso wie Sachsen betonen, dass die Braunkohle mittelfristig weiterhin hohe Bedeutung haben soll, forcieren sie gleichzeitig den Ausbau der erneuerbaren Energien. Während die Bundesregierung bis 2020 einen Anteil der erneuerbaren Energien von $35 \%$ an der Stromerzeugung anvisiert, streben insbesondere Brandenburg und Sachsen-Anhalt deutlich höhere Ziele an. Brandenburg möchte bis 2020 Stromexportland allein durch die Stromerzeugung bei den erneuerbaren Energien werden und auch Sachsen-Anhalt kommt mit den vorgesehenen $80 \%$ einer rechnerischen Vollversorgung durch erneuerbare Energien nahe (vgl. Breyer/Müller 2013). Diese energiewirtschaftliche Doppelstrategie schlägt sich aktuell auch in der ökonomischen Bedeutung von Braunkohlewirtschaft und erneuerbaren Energien nieder. Die Arbeitsplätze in der Braunkohlewirtschaft konnten sich in Brandenburg, Sach-

\footnotetext{
${ }^{4}$ Zwar hat das Kabinett der aktuellen Landesregierung im April 2015 Grundzüge eines ambitionierten Klimaschutzplans beschlossen, den Schwerpunkt bildet aber weiterhin die Modernisierung und Effizienzerhöhung bei Kohlekraftwerken. Bis zum Jahr 2025 soll der Anteil der erneuerbaren Energien von derzeit 11 auf 30 \% vor allem durch RePowering von Windenergie, nicht aber durch neue Flächenausweisungen erhöht werden. Nordrhein-Westfalen liegt damit weiterhin deutlich unter dem Bundesdurchschnitt.
} 
Tab. 1 Bruttobeschäftigung durch erneuerbare Energien (EE) in den Bundesländern. (nach Ulrich/Lehr 2014: 7)

\begin{tabular}{lrr}
\hline Bundesland & Bruttobeschäftigung EE (2013) & Bruttobeschäftigung EE je 1000 Beschäftigte (2013) \\
\hline 1. Sachsen-Anhalt & 24.320 & 26,8 \\
2. Mecklenburg-Vorpommern & 14.980 & 23,2 \\
3. Brandenburg & 17.580 & 18,8 \\
4. Niedersachsen & 55.200 & 16 \\
5. Bremen & 5510 & 14,4 \\
6. Schleswig-Holstein & 15.740 & 13,7 \\
7. Thüringen & 11.460 & 12,5 \\
8. Bayern & 60.540 & 9,8 \\
9. Sachsen-Anhalt & 16.400 & 9,3 \\
10. Hamburg & 9010 & 8,6 \\
11. Baden-Württemberg & 40.540 & 7,7 \\
12. Rheinland-Pfalz & 12.610 & 7,3 \\
13. Hessen & 20.160 & 6,9 \\
14. Nordrhein-Westfalen & 50.330 & 6,2 \\
15. Saarland & 2650 & 5,6 \\
16. Berlin & 6070 & 3,9 \\
Deutschland & 363.100 & 9,7 \\
\hline
\end{tabular}

Unter Bruttobeschäftigung werden die Arbeitsplatzeffekte durch erneuerbare Energien in den Bereichen Anlagenproduktion, Betrieb und Wartung, Bereitstellung von Biomasse (inklusive Vorleisterindustrien) sowie Forschung und öffentliche Verwaltung verstanden (vgl. Ulrich/Lehr 2014: 6). Zum Vergleich und zur Einordnung der Dimension der Bruttobeschäftigung durch erneuerbare Energien können die Arbeitsplätze im Kohleabbau herangezogen werden. Diese lagen im Jahr 2014 bei insgesamt (Stein- und Braunkohle) 33.500 Arbeitsplätzen (vgl. Statistik der Kohlenwirtschaft e. V. 2015)

sen und Sachsen-Anhalt auf dem Niveau von Mitte der 1990er-Jahre stabilisieren bzw. sogar leicht erhöhen (vgl. Prognos 2011: 10). Während die erneuerbaren Energien in Sachsen hinsichtlich des Arbeitsplatzniveaus nur in etwa einen bundesdurchschnittlichen Wert erreichen, haben die erneuerbaren Energien in Brandenburg und Sachsen-Anhalt inzwischen eine sehr hohe Bedeutung für den regionalen Arbeitsmarkt (vgl. Tab. 1).

Auf der Seite der Bundesländer, die sich durch eine deutlich weniger karbonintensive Wirtschaftsstruktur auszeichnen, zeigt sich ein sehr differenziertes Bild. Ein wichtiges Unterscheidungsmerkmal für die jeweilige Positionierung in der nationalen Klimapolitik ist dabei zunächst die Rolle der Kernenergie. Kernkraftwerke wurden in Deutschland hauptsächlich in den drei südlichen großen Bundesländern sowie in Niedersachsen und Schleswig-Holstein errichtet. Unionsgeführte Landesregierungen ${ }^{5}$ forderten nach dem ersten Atomausstiegsbeschluss der rot-grünen Bundesregierung von 2001 durchweg in den Klimaprogrammen eine Laufzeitverlängerung für Kernkraftwerke. Die Klimaprogramme insbesondere der drei südlichen großen Flächenländer sind von Beginn an davon geprägt, dass sie

\footnotetext{
5 Seit den Landtagswahlen in Hessen 1999 hatten alle drei südlichen großen Bundesländer und Niedersachsen eine CDU- oder CSU-geführte Landesregierung. In Schleswig-Holstein stellte die CDU von 2005 bis 2012 den Ministerpräsidenten, eine explizit kernkraftfreundliche Position wurde aber erst nach dem Ende der dortigen großen Koalition (2005-2009) unter der CDU-FDP-Regierung vertreten.
}

wie Baden-Württemberg und Bayern den landeseigenen Spielraum für Emissionsminderungen aufgrund des vergleichsweise geringen Emissionsniveaus pro Einwohner nur als sehr gering einschätzen oder wie Hessen sogar leicht steigende Emissionen als unkritisch ansehen. Neben der Formulierung von relativ wenig ambitionierten $\mathrm{CO}_{2}$ Reduktionszielen zeichneten sich ihre Klimaprogramme insbesondere auch durch die klare Ablehnung des Ausbaus der Windenergie mit Verweis auf deren „Unstetigkeit“ und den damit verbundenen hohen Kosten zur Gewährleistung der Versorgungssicherheit aus. ${ }^{6}$ Damit unterscheiden sie sich klar von den beiden nördlichen Bundesländern mit einem hohen Kernkraftanteil. Unabhängig von der Zusammensetzung der Landesregierungen haben Niedersachsen und Schleswig-Holstein seit den 1990er-Jahren als Vorreiter beim Windenergieausbau und als dessen regionalwirtschaftliche Profiteure stets den ambitionierten Ausbau befürwortet (Ulrich/Lehr 2014: 9).

Seit der Reaktorkatastrophe 2011 in Fukushima wird der Ausstieg aus der Kernenergienutzung sowie der Ausbau der Windenergie auch von unionsgeführten Landesregierungen unterstützt, in Baden-Württemberg zeigen sich seit

\footnotetext{
${ }^{6}$ Vergleichende Analysen der raumplanerischen Rahmenbedingungen in den Bundesländern erklären die ablehnenden Haltungen dieser Bundesländer weniger mit geographisch bedingten schlechteren Erzeugungspotenzialen, sondern vor allem mit politischen Motiven (vgl. Ehlers/Böhme 2011: 325 ff.).
} 


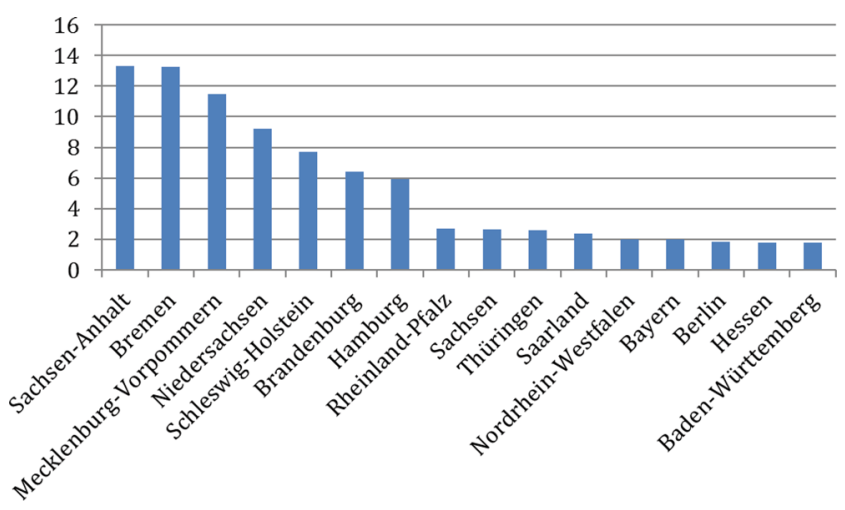

Abb. 3 Bruttobeschäftigung Windenergie in 2013 je 1000 Beschäftigte in den Bundesländern. (nach Ulrich/Lehr 2014: 9)

der Regierungsübernahme durch die grün-rote Landesregierung (2011) ferner neue ambitionierte Schwerpunktsetzungen bei der Systemintegration erneuerbarer Energien (vgl. Smart Grids Plattform Baden-Württemberg 2013). Dagegen macht Bayern durch die 2013 erfolgreich initiierte Länderöffnungsklausel zur Vorgabe von Abstandsregelungen bei Windenergieanlagen und den aktuellen Widerstand gegen den Ausbau der Stromnetztrasse „Süd-Link“ deutlich, dass ein umfassender Ausbau der Windenergie politisch nicht unterstützt wird.

Die übrigen Bundesländer, in denen die Kohle- und Nuklearwirtschaft relativ unbedeutend sind, zeichnen sich durch eher ambitionierte Klimaziele aus. So unterstützen sowohl die Stadtstaaten als auch Rheinland-Pfalz, Thüringen und Mecklenburg-Vorpommern in ihren Klimaschutzund Energieprogrammen die nationalen Klimaschutzziele und streben eine Emissionsminderung in gleicher Größenordnung an. Ähnlich wie das schon skizzierte Bundesland Schleswig-Holstein versucht Mecklenburg-Vorpommern sich weiterhin durch den Ausbau der Windenergie zu profilieren. Seit Anfang der 2000er-Jahre haben die dortigen Landesregierungen versucht, die Windenergie im Bundesland stark auszubauen und wichtige Standorte der industriellen Fertigung von erneuerbaren Energien anzusiedeln. Wie in den anderen Küstenländern und auch in den Hansestädten ist die Bedeutung der Windenergie für die regionale Beschäftigung in Mecklenburg-Vorpommern sehr hoch (siehe Abb. 3).

Deutlich wird, dass im Hinblick auf die klimapolitischen Ziele der ökonomische Anpassungsdruck zur Reduktion von $\mathrm{CO}_{2}$-Emissionen und die ökonomischen Entwicklungspotenziale durch Ausbau der erneuerbaren Energien in vielen Fällen regional stark auseinanderfallen und klare räumliche Umverteilungseffekte und damit verbunden auch deutliche subnationale Interessenunterschiede in der Klimapolitik zu erwarten sind.

Im Vergleich beider staatlicher Entscheidungsebenen bleiben die Bundesländer bei der Formulierung ihrer Kli- maschutzziele dabei durchweg deutlich unter dem Niveau des nationalen Ziels. In der vom Umweltbundesamt herausgegebenen Studie von 2011 zeigt sich für die damals existierenden Klimaschutzziele der Bundesländer, dass diese in der Summe für 2020 gegenüber 1990 nur auf eine $\mathrm{CO}_{2}$-Minderung von 26,7 \% hinauslaufen, während der Bund eine Minderung um $40 \%$ anvisiert (Biedermann 2011: 62). Auch unter Berücksichtigung der seitdem neu gefassten Klimaschutzziele der Bundesländer ergeben sich nur leichte Veränderungen. Dagegen zeichnen sich die Aussagen der Bundesländer zum anvisierten Ausbau der erneuerbaren Energien durch eine kontinuierliche Konkretisierung und Steigerung der Zielsetzungen aus. Aktuell übertreffen sie in der Summe die nationalen Zielsetzungen fast um den Faktor 2 und visieren einen Anteil der erneuerbaren Energien am Stromverbrauch von $68 \%$ bis 2020 an (Bund $35 \%$ ) (vgl. Breyer/Müller 2013: 17). Diese Diskrepanz kann dadurch erklärt werden, dass die $\mathrm{CO}_{2-}$ Minderung für klassische Industriezweige zunächst hohe Kosten bewirken kann, während der Ausbau erneuerbarer Energien zeitweise parallel zur fossilen Energieerzeugung verfolgt werden kann und zusätzliche ökonomische Potenziale für diejenigen Regionen und Länder bietet, die früh aktiv werden.

\section{Föderale Verhandlungslogiken der Energiewendepolitik}

Im Unterschied zu den europäischen Regelungen zur Lastenverteilung der vorgesehenen Emissionsminderungen unter den Mitgliedsländern ('burden sharing'), die das europäische Emissionsminderungsziel durch nationale Emissionsziele (und Ziele im Bereich der erneuerbaren Energien) konkretisieren, wurde in Deutschland auf explizite Verteilungsregelungen verzichtet. Stattdessen werden die klimapolitischen Ziele der Bundesregierung seit dem ersten nationalen Klimaschutzprogramm durch die Definition von Zielen und Maßnahmenpaketen für die relevanten Sektoren der Industrie, Energiewirtschaft, Haushalte, Verkehr sowie Land- und Forstwirtschaft realisiert (vgl. Agora Energiewende 2015: 27). Dagegen wird in den bislang sechs Berichten der Interministeriellen Arbeitsgruppe „, $\mathrm{CO}_{2}$-Reduktion“ allenfalls am Rande auf die Bedeutung der Bundesländer für die Realisierung der nationalen Klimaziele eingegangen. Weder in den nationalen Klimaschutzprogrammen noch in der Umweltministerkonferenz der Bundesländer bzw. in der Bund-Länder-Arbeitsgemeinschaft „Klima, Energie, Mobilität - Nachhaltigkeit“" wurde über Vereinbarungen verhandelt, wie das nationale Emissionsziel oder die Ziele im Bereich der Stromproduktion auf der Basis erneuerbarer Energien auf die einzelnen Bundesländer verteilt werden. Zugleich existieren keine prozeduralen Regelun- 
gen des Bundes, welche die Bundesländer zur Aufstellung eigenständiger Klimaschutzprogramme verpflichten. Stattdessen erfolgt die räumliche Allokation der Kosten und Nutzen im Klimaschutz implizit und räumlich nicht koordiniert über Bundesregelungen, über die in föderalen Verhandlungssystemen entschieden wird. Im Folgenden werden die Logiken und Ergebnisse der föderalen Verhandlungssysteme am Beispiel der Förderung der erneuerbaren Energien und des Emissionshandels untersucht.

\subsection{Die Förderung erneuerbarer Energien}

Während die nationale Politik lange Zeit jenseits von Forschungs- und Entwicklungsförderung im Bereich erneuerbarer Energien wenig aktiv wurde, waren es insbesondere einzelne Bundesländer, die Fördermaßnahmen initiierten. $\mathrm{Zu}$ den Vorreitern zählten hier insbesondere NordrheinWestfalen, aber auch Hessen, Niedersachsen und Schleswig-Holstein (vgl. Suck 2008). Erst im Jahr 1991 wurde mit dem Stromeinspeisungsgesetz ein einheitlicher rechtlicher Rahmen geschaffen. Dieser basierte vor allem auf Erfahrungen einzelner Bundesländer wie Nordrhein-Westfalen, Niedersachsen oder Schleswig-Holstein (Suck 2008: $116,161)$ und bewirkte in den 1990er-Jahren ein maßvolles Wachstum der Windenergie, Biomasse und Wasserkraft in vielen Fällen unterstützt durch komplementäre Förderprogramme der Länder.

Ende der 1990er-Jahre stellten sich zwei Herausforderungen: Einerseits sanken im Zuge der Liberalisierung die Strompreise zumindest moderat und Investitionen in die Energieinfrastruktur wurden unsicherer. Die erneuerbare Energiewirtschaft forderte daher höhere Vergütungssätze und eine stärkere Entkopplung der Einspeisevergütung von den Marktpreisen (Michaelowa 2005: 195). Andererseits führte der vergleichsweise hohe Anteil der Windenergie in den Küstenregionen zu hohen Kosten der betroffenen Netzbetreiber (Suck 2005: 171). Das mit den hohen Vergütungssätzen verbundene Szenario von einer räumlich stark ungleichen Entwicklung der Strompreise wurde als Standortnachteil insbesondere für die ansässigen energieintensiven Industrien betrachtet. Infolgedessen forderten die nördlichen Bundesländer eine angemessene Lösung (Suck 2008: 328).

Die Verabschiedung des Erneuerbare-Energien-Gesetzes (EEG) durch die rot-grüne Bundesregierung im Jahr 2000 kann daher auch als Reaktion auf beide Herausforderungen gesehen werden. So wurden mit der Reform nicht nur deutlich höhere Vergütungssätze für die verschiedenen Sparten der erneuerbaren Energien eingeführt, welche den Betreibern langfristige Investitionssicherheit unabhängig von der allgemeinen Marktentwicklung garantierten. Ferner sicherte ein nationaler Umlagemechanismus auf der Ebene der Übertragungsnetzbetreiber eine Verteilung der Kosten auf die Energieverbraucher im gesamten Bundesgebiet. Indem die Kosten der erneuerbaren Stromerzeugung damit gewissermaßen ,enträumlicht“ wurden, die potenziellen Nutzen der erneuerbaren Energiewirtschaft in Form von Investitionen, Arbeitsplätzen und Steuereinnahmen jedoch regional wirksam wurden, wurden mit der Einführung des EEG nicht nur die allgemeinen Investitionsanreize erhöht, sondern auch ein Mechanismus zur interregionalen Konkurrenz um die hiermit ausgelösten Vergütungen etabliert.

Bei den Beratungen zum Erneuerbaren-Energien-Gesetz wurde deutlich, dass das EEG zum Gegenstand umfangreicher Kontroversen der politischen Parteien wurde. Während das Stromeinspeisungsgesetz noch mit einer parteienübergreifenden Mehrheit verabschiedet wurde, musste das EEG von der Regierungskoalition gegen erhebliche Widerstände der konservativ-liberalen Opposition durchgesetzt werden. Da die Regierungsparteien im zustimmungspflichtigen Bundesrat jedoch keine Mehrheit hatten, drohte das Gesetz zu scheitern. Allerdings kamen regionalwirtschaftliche Interessen zum Tragen und die Vertreter des konservativ regierten Thüringen stimmten dem Vorhaben zur Unterstützung ihrer regionalen Agrarwirtschaft zu, die die Nutzung der Biomasse ausbauen wollten. Regionalwirtschaftliche Interessen wurden auch bei der Integration einer Härtefallregelung wirksam, um die das EEG im Jahr 2003 ergänzt wurde. So setzte der ehemalige Ministerpräsident von Nordrhein-Westfalen und amtierende Bundeswirtschaftsminister durch, dass 560 energieintensive Industriekunden, die in der Mehrzahl in Nordrhein-Westfalen ansässig waren, von der EEG-Umlage befreit wurden (Jacobsson/Lauber 2006: 269).

Anders als der „Abweichler“ Thüringen verstärkten die konservativ regierten West-Bundesländer ihre Kritik am EEG. Insbesondere die Kritik der Landesregierungen von Baden-Württemberg, Hessen und Bayern richtete sich hierbei vor allem auf den Windenergieausbau zulasten des Natur- und Landschaftsschutzes. Durch die Ausschöpfung ihrer planungsrechtlichen Handlungsspielräume schufen sie nicht nur umfangreiche Restriktionen für die Installation von Windenergieanlagen, ${ }^{7}$ sondern erreichten über ihre Bundesratsmehrheit auch eine Reduzierung der Einspeisevergütung für Windenergie (Hirschl 2008: 162). Während diese Kombination aus raumplanerischen Restriktionen und reduzierten Vergütungssätzen den Ausbau der Windenergie zwar verlangsamte (Büsgen/Dürrschmidt 2009: 2541),

\footnotetext{
7 So verpflichtete das 2003 novellierte Landesplanungsgesetz in Baden-Württemberg die regionalen Planungsträger, flächendeckend Vorranggebiete für die Windkraftnutzung mit Ausschlusswirkung für die übrigen Gebiete auszuweisen (sog. Schwarz-Weiß-Regelung). Ähnlich agierten bis 2011 auch die regionalen Planungsträger in Hessen und Bayern. $\mathrm{Zu}$ den restriktiven Wirkungen dieser regionalplanerischen Regelungen vgl. Ehlers/Böhme 2011: 326.
} 


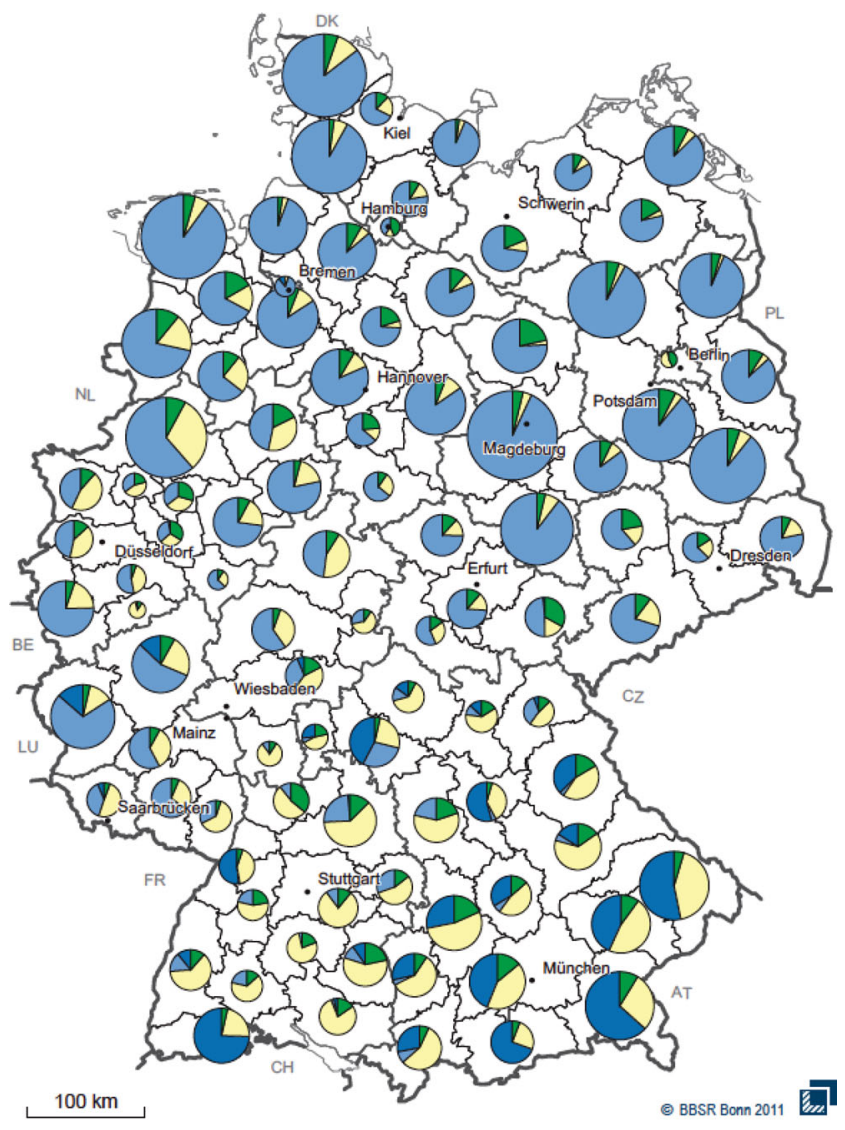

Installierte elektrische Leistung erneuerbarer Energieträger 2010 in Megawatt

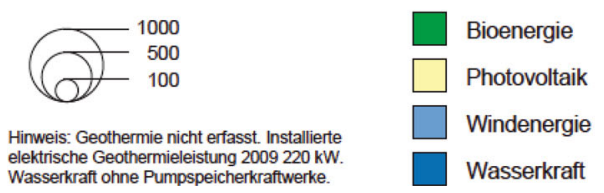

Abb. 4 Regionale Verteilung der erneuerbaren Stromproduktion 2010. (BBSR 2012: 94)

setzte sich das rapide Wachstum der erneuerbaren Energien insgesamt fort.

Im Unterschied zur starken Kritik an der kontinuierlich wachsenden EEG-Umlage durch die konservativen und liberalen Bundesparteien und Landesregierungen der Südländer waren die deutlich höheren Vergütungssätze für die Fotovoltaik kaum Gegenstand von Kritik, da besonders Bayern und Baden-Württemberg von günstigen Standortbedingungen für die Fotovoltaik (und Biomasse) profitierten (siehe Abb. 4). Insbesondere die ansässigen Hauseigentümer, Handwerksbetriebe und die Landwirtschaft profitierten von der Solarförderung des EEG, sodass Bayern in den Folgejahren zum Hauptnutznießer der EEG-Finanzströme wurde, wenngleich sein Gesamtbeitrag zur erneuerbaren Stromerzeugung unterproportional war (siehe Abb. 5). Auch andere CDU-regierte Länder (Sachsen, Thüringen, SachsenAnhalt) profitierten von der Förderung aufgrund der in die- sen Ländern ansässigen Anlagenhersteller. Dagegen zählt Nordrhein-Westfalen zu den klaren Verlierern bei der Allokation der EEG-Zahlungsströme (vgl. Gawel/Korte 2015).

Aufgrund der regionalwirtschaftlichen Interessen konnte auch die zwischen 2009 und 2013 amtierende konservativ-liberale Bunderegierung entgegen den Programmatiken der FDP- und CDU-Bundesparteien keine Einigung mit den konservativ regierten Bundesländern über eine strukturelle Reform des EEG erzielen. Bedingt durch die heterogene Interessenlage in den konservativen Regierungskreisen konnte keine Bundesratsmehrheit für eine grundlegende EEGReform gefunden werden, welche die EEG-Umlage und die dadurch verursachten Kostensteigerungen begrenzt hätte. Die regionalwirtschaftlichen Interessen am Fortbestand der EEG-Förderung, die damit verbundenen föderalen Dynamiken in der Bund-Länder-Abstimmung, aber auch die durch das EEG ausgelösten interregionalen Wettbewerbseffekte ermöglichten Deutschlands internationale Vorreiterrolle im Bereich der erneuerbaren Energien. So stieg ihr Anteil an der Stromversorgung von 6,2 \% im Jahr 2000 auf 27,3 \% im Jahr 2014 (Agora Energiewende 2015). Im Jahr 2013 arbeiteten 371.000 Beschäftigte in der erneuerbaren Energiewirtschaft (O'Sullivan/Edler/Bickel et al. 2014: 12).

Allerdings geriet das EEG zunehmend in die Kritik der europäischen Kommission, die insbesondere in den Ausnahmeregelungen für energieintensive Unternehmen von der EEG-Umlage einen Verstoß gegen europäisches Wettbewerbsrecht und eine unzulässige Beihilfe sah. Durch die kontinuierliche Ausweitung dieser Ausnahmeregelungen beliefen sich die Entlastungen für größere Industrieunternehmen im Jahr 2014 auf ca. 5,1 Milliarden Euro (BMWI 2015: 23). Zudem wurde das durch das EEG induzierte Umlagevolumen und die damit verbundene Belastung der Haushalte und kleinen und mittelständischen Unternehmen auch im öffentlichen Mediendiskurs zunehmend kontrovers diskutiert und traf auf wachsende politische Widerstände. Durch den Anstieg der EEG-Umlage von 1,5 Milliarden Euro im Jahr 2000 auf 23,6 Milliarden Euro in 2014 (BDEW 2014: 47) kam es zu einem kontinuierlichen Anstieg der Strompreise (siehe Abb. 6) und die Finanztransfers wurden zunehmend auch als Problem des Schutzes ökonomisch benachteiligter Verbraucher diskutiert. Ferner wurde kritisiert, dass insbesondere die Fotovoltaik mit ihrem Anteil von $19 \%$ an der erneuerbaren Stromgewinnung $48 \%$ der durch das EEG induzierten Finanzmittel erhielt, während die deutlich günstigere Onshore-Windenergie lediglich $19 \%$ der Umlage erhielt, aber einen Anteil von $33 \%$ an der Stromproduktion hatte (BDEW 2014: 11, 48). Nicht zuletzt wurde der mit dem Wachstum der erneuerbaren Energien verbundene Umverteilungsmechanismus auch zunehmend zu einem föderalen Konfliktfeld: Während Bayern als zentraler Nutznießer eine positive Zahlungsbilanz von 0,8 Milliarden Euro hatte, beliefen sich die Finanztrans- 
Abb. 5 Regionale Verteilung der EEG-Stromerzeugung und Salden der gesamten EEGZahlungsströme (in Mrd. Euro) 2010-2013. (BDEW 2014)

\begin{tabular}{|c|c|c|c|}
\hline Bundesland & $\begin{array}{l}\text { Anteil an EEG- } \\
\text { Stromerzeugung }\end{array}$ & $\begin{array}{c}\text { Anteil an EEG- } \\
\text { Vergütung }\end{array}$ & 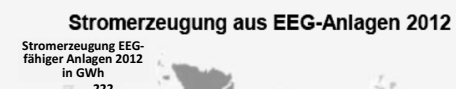 \\
\hline Baden-Württemberg & $8,0 \%$ & $11,5 \%$ & \\
\hline Bayern & $16,4 \%$ & $24,9 \%$ & \\
\hline Berlin & $0,2 \%$ & $0,2 \%$ & \\
\hline Brandenburg & $9,9 \%$ & $6,9 \%$ & \\
\hline Bremen & $0,3 \%$ & $0,2 \%$ & \\
\hline Hamburg & $0,2 \%$ & $0,2 \%$ & \\
\hline Hessen & $3,1 \%$ & $3,7 \%$ & \\
\hline $\begin{array}{l}\text { Mecklenburg- } \\
\text { Vorpommern }\end{array}$ & $4,8 \%$ & $3,8 \%$ & \\
\hline Niedersachsen & $18,4 \%$ & $15,6 \%$ & \\
\hline Nordrhein-Westfalen & $10,5 \%$ & $10,0 \%$ & \\
\hline Rheinland-Pfalz & $4,1 \%$ & $3,9 \%$ & \\
\hline Saarland & $0,8 \%$ & $0,6 \%$ & \\
\hline Sachsen & $3,5 \%$ & $3,4 \%$ & \\
\hline Sachsen-Anhalt & $7,7 \%$ & $5,3 \%$ & \\
\hline Schleswig-Holstein & $8,5 \%$ & $6,8 \%$ & \\
\hline Thüringen & $2,9 \%$ & $2,5 \%$ & \\
\hline Offshore Wind-Gebiete & $0,6 \%$ & $0,5 \%$ & \\
\hline
\end{tabular}

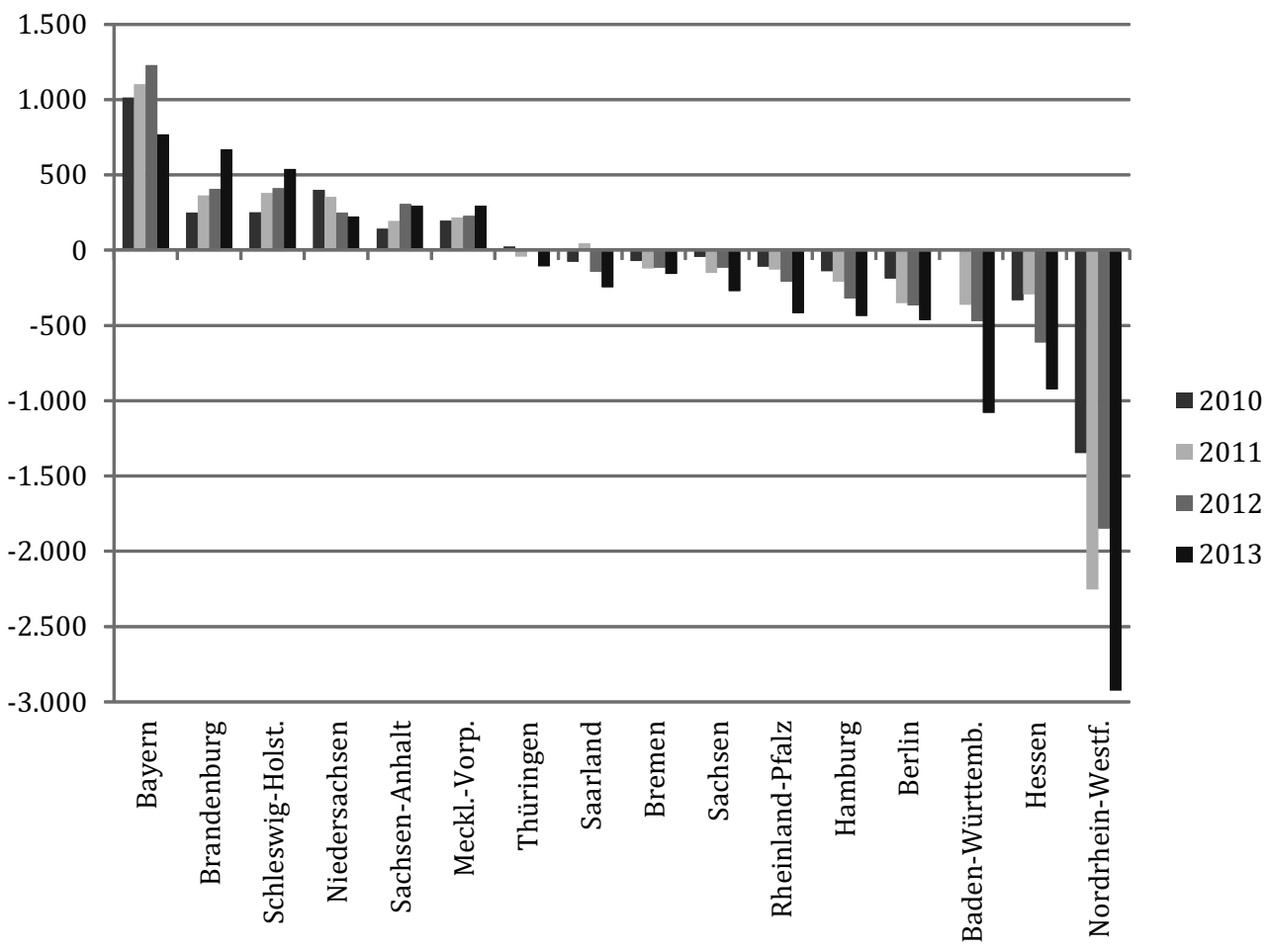

fers der Energieverbraucher in Nordrhein-Westfalen an Anlagenbetreiber in anderen Bundesländern auf insgesamt 2,9 Milliarden Euro (BDEW 2014: 84), was etwa der vierfachen Summe der Zahlungen an Nordrhein-Westfalen aus dem Länderfinanzausgleich entspricht.

Mit der Regierungsübernahme durch eine zweite große Koalition Ende des Jahres 2013 im Bund wurden daher auch umfassende Strukturreformen des EEG angekündigt. In einem ersten Reformschritt (dem sogenannten „EEG 2.0“), der im August 2014 rechtskräftig wurde, wird ein Ausbaukorridor der erneuerbaren Energien gesetzlich festgelegt (40-45 \% im Jahr 2025, 55-60 \% im Jahr 2035). Zugleich wurden flexible (,,atmende“) Obergrenzen für jede Sparte der erneuerbaren Energien mit dem Ziel eingeführt, primär die kosteneffizientesten Energietechnologien zu fördern. Der Anstieg der EEG-Umlage soll durch eine weitere Degression der Einspeisevergütungen reduziert und die Ausnahmeregelungen für energieintensive Industrien ein- 
Abb. 6 Entwicklung der EEGUmlage (ct/kWh) für private Haushalte in den Jahren 2000 bis 2014. (BMU 2013)

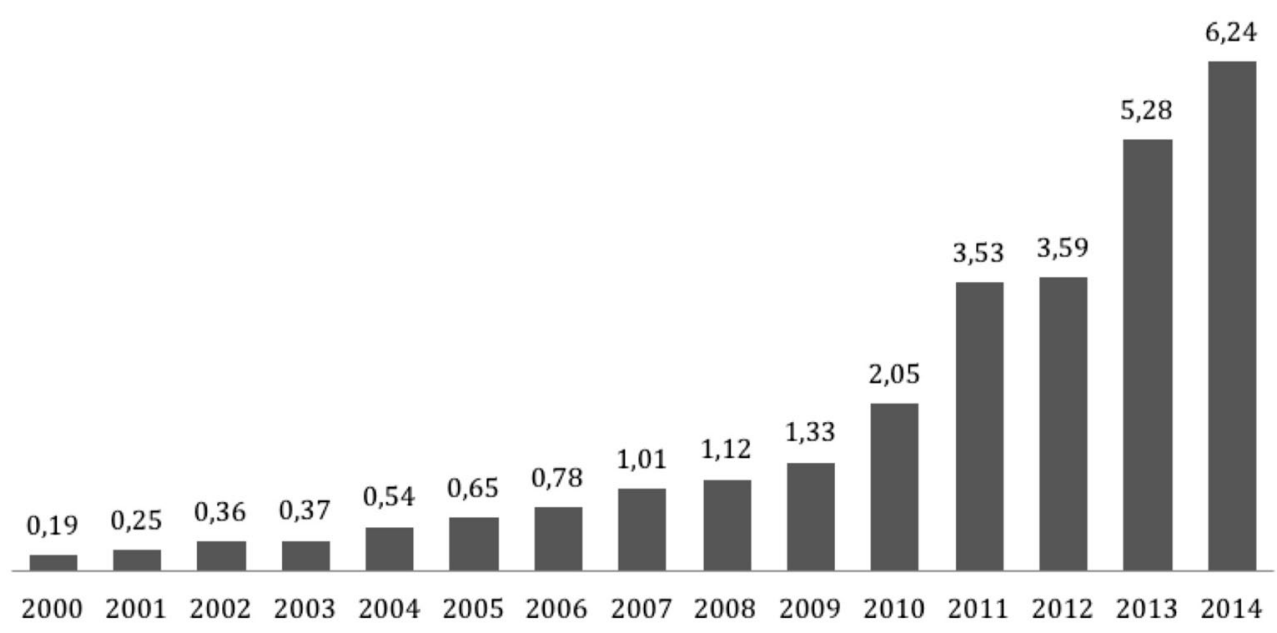

geschränkt werden. In einem zweiten, für das Jahr 2017 angekündigten Reformschritt (,EEG 3.0“) soll die Förderhöhe der erneuerbaren Energien nicht länger durch eine gesetzlich festgelegte Einspeisevergütung, sondern durch die Einführung von Ausschreibungsmodellen definiert werden.

Die Reformdiskussionen lösten insbesondere auf Seiten der Bundesländer umfangreiche Kontroversen und Konflikte aus, bei denen etablierte parteipolitische Positionen in den Hintergrund traten. Zahlreiche Landesregierungen verschafften ihren regionalwirtschaftlichen Interessen über den Bundesrat bzw. über den seit dem Jahr 2006 regelmäBig tagenden Energiegipfel im Kanzleramt offensiv Nachdruck: So setzte sich Nordrhein-Westfalen für die maximale Beibehaltung der Ausnahmeregelungen für energieintensive Industrien ein, die „Küstenländer“ engagierten sich für großzügigere Ausbaukorridore und höhere Fördersätze bei der Windenergie. Sie wurden darin von RheinlandPfalz und Baden-Württemberg unterstützt, die nach dem Wechsel der Landesregierungen im Jahr 2011 ambitionierte Planungen zum Ausbau der Onshore-Windenergie umsetzen wollten, während sich Bayern und Thüringen für die Förderung der Biomasse (und Fotovoltaik) stark machten. Dementsprechend wurden den Länderregierungen auf dem Energiegipfel zahlreiche Zugeständnisse gemacht. So wurde der Schritt zu einem auktionsbasierten Marktdesign erst für das Jahr 2017 angekündigt und die Obergrenzen für alle Sparten der erneuerbaren Energien entsprechend ihrer jeweiligen Wachstumspfade in den Vorjahren großzügiger bemessen. ${ }^{8}$ Allerdings traf die auf dem Energiegipfel erzielte Vereinbarung zwischen Bund und Ländern auf wettbewerbsrechtliche Bedenken der Europäischen Kommission. In der Folge mussten die Ausnahmeregelungen für industri-

\footnotetext{
${ }^{8}$ Darüber hinaus wird eine verpflichtende Direktvermarktung für Neuanlagen ab 2017 (bis dahin nur für Neuanlagen ab 5 MW) eingeführt, womit Strom aus erneuerbaren Energiequellen künftig an Großabnehmer oder an der Strombörse verkauft werden muss.
}

elle Verbraucher und industrielle Eigenerzeugung reduziert werden. Zudem werden die Ausschreibungsmodelle schrittweise für europäische Bieter geöffnet.

Zusammenfassend zeigt sich, dass die Überlagerung von Interessenkonstellationen im parteipolitischen Wettbewerb mit regionalwirtschaftlichen Standortinteressen den Ausbau erneuerbarer Energien eher unterstützt und den mit der Verabschiedung des Erneuerbaren-Energien-Gesetzes 2000 eingeschlagenen Weg des ambitionierten Ausbaus erneuerbarer Energien stabilisiert hat. Jenseits inkrementeller Reformen der Bundesregierung haben die regionalwirtschaftlichen und politischen Interessen der Bundesländer seitdem einen grundsätzlichen Richtungswechsel in der Energiepolitik verhindert. Neben einem grundsätzlichen parteienübergreifenden Konsens lag ein wesentlicher Grund darin, dass die Kosten für die Förderung der erneuerbaren Energien direkt von den Verbrauchern getragen werden. Seit Einführung des EEG wurden die Standortvorteile für die erneuerbare Energiewirtschaft und den Anlagenbau zu wichtigen Bestandteilen der Umwelt- und Wirtschaftspolitiken der Länder. Durch die Externalisierung der Kosten an die Verbraucher, ${ }^{9}$ aber auch weil die räumlichen Umverteilungswirkungen zum Zeitpunkt der Einführung des EEG unterschätzt wurden, konnten föderale Entscheidungsblockaden vermieden werden. Entgegen den Befunden der Föderalismusforschung konnte durch die institutionellen Mechanismen des EEG ein föderaler Wettbewerb um die Teilhabe an den durch das EEG ausgelösten Finanzflüssen ge-

\footnotetext{
${ }^{9}$ Mit dem Begriff der Kostenexternalisierung soll zum Ausdruck gebracht werden, dass die Kosten der Energiewende zu einem überproportionalen Anteil von den Privathaushalten bzw. zu einem unterproportionalen Anteil von den großen industriellen Verbrauchern mit stärkerer Lobby in den Bund-Länder-Verhandlungen getragen werden. Betont werden soll jedoch, dass es sich bei dieser „Kostenexternalisierung“ gleichzeitig um eine Internalisierung von Umweltkosten handelt - wenngleich ungleich verteilt und nur eingeschränkt dem Verursacherprinzip Rechnung tragend.
} 
fördert werden. Während die EU-Kommission lange Zeit kaum in die deutsche Förderpolitik interveniert hat, hat diese in jüngerer Zeit zunehmend Einfluss auf die föderale Politikgestaltung genommen, wodurch die Ausnahmeregelungen begrenzt und die regenerativen Energiemärkte teilweise für europäische Bieter geöffnet wurden.

\subsection{Die Gestaltung des europäischen Emissionshandelssystems}

Mit der Einführung eines europaweiten $\mathrm{CO}_{2}$-Emissionshandelssystems (EHS) 2003/2004 wurde ein Instrument in die deutsche Klimapolitik integriert, welches deutlich stärker als der bis dahin verfolgte nationale Ansatz den Einsatz ökonomischer Regelungsmechanismen betont. Grundsätzliche Vorbehalte bei der Einführung des Emissionshandels existierten dabei sowohl auf Bundes- als auch auf Bundesländerebene (Ziesing 2009: 348-349). Der Bundeswirtschaftsminister (und ehemalige nordrhein-westfälische Ministerpräsident) scheiterte jedoch auf EU-Ebene damit, die Einführung des Emissionshandels zu verhindern (Skjaerseth/Wettestad 2008: 109-115).

Anschließend entzündeten sich die Konflikte zunächst an der Kompetenzverteilung zwischen den Bundesländern und der Bundesregierung. Ähnlich wie bei der Umsetzung des Bundesimmissionsschutzgesetzes (BImSchG) durch die Bundesländer beanspruchten diese die uneingeschränkten Vollzugskompetenzen im Emissionshandel. Dagegen bevorzugte das federführende Bundesministerium für Umwelt, Naturschutz und Reaktorsicherheit (BMU) eine geteilte $\mathrm{Zu}$ ständigkeit, aber mit dem Vorbehalt, die strategisch wichtigsten Aufgaben (z. B. technische Verwaltung des Handelssystems, Zuteilung von Zertifikaten, Überwachung, Sanktionierung) auf Bundesebene anzusiedeln (Bundesrat 2004: 163; Hartmann 2004: 83). Die Bundesregierung bevorzugte eine Zentralisierung und Unitarisierung von Vollzugsaufgaben, da eine Gleichbehandlung aller Kraftwerksbetreiber unabhängig von regionalen wirtschaftlichen oder politischen Interessen aufgrund der komplexen behördlichen Genehmigungsverfahren in jedem Bundesland schwer zu gewährleisten und zu überwachen gewesen wäre.

Da durch das Treibhausgasemissionshandelsgesetz $(\mathrm{TEHG})^{10}$ Belange der Bundesländer betroffen waren, benötigte die Bundesregierung die Zustimmung des Bundesrates, in dem die konservativ-liberale Opposition mit ihrer Stimmenmehrheit in die Verhandlungen über das

\footnotetext{
10 Neben der formalen Einführung des EU-Emissionshandelssystems EHS in das deutsche Recht war nur die Definition von Kompetenzen das Schlüsselthema des TEHG, das am 15. Juli 2004 in Kraft gesetzt wurde (Hartmann 2004: 75). Alle für den Staatshaushalt relevanten Fragen bezüglich der Zertifikate und die Regeln der Zuteilung wurden in den Nationalen Allokationsplan (NAP) verlagert, welcher im Oktober 2004 in Kraft trat.
}

TEHG intervenierte. Am Ende war die Bundesregierung in der Lage, ihre Präferenz der geteilten Zuständigkeit durchzusetzen, indem sie den Bundesländern mit der Durchsetzung ausschließlich auf Bundesebene drohte (Bundesrat 2004: 163; Hartmann 2004: 110). Dies hätte umfangreiche Doppelzulassungen für die betroffenen Anlagen erfordert (nach TEHG auf Bundesebene und nach BImSchG auf Länderebene). Mit doppelten Genehmigungsverfahren konfrontiert, intervenierte die Industrie und drängte die Bundesländer dazu, einer geteilten Zuständigkeit zuzustimmen (Hartmann 2004: 110). Zusätzlich waren die kleineren Bundesländer für eine nationale Regelung. Im Gegensatz zu den größeren Bundesländern hatten sie nur begrenzte Verwaltungsmittel und widersprachen aus diesem Grund nicht der zentralen Administration auf Bundesebene. Demgegenüber hätte Nordrhein-Westfalen genügend Kapazität gehabt, um die Vollzugsaufgaben zu bewältigen und positionierte sich trotz einer ebenfalls rot-grünen Landesregierung gegen die Bundesregierung. Obwohl NordrheinWestfalen gemeinsam mit den anderen großen, von konservativen und liberalen Parteien regierten Bundesländern zwei Drittel der Bevölkerung und $90 \%$ der stark betroffenen Industrie vertraten, waren sie schließlich nicht in der Lage, genügend kleinere Bundesländer zu überzeugen, und gewannen daher keine Mehrheit im Bundesrat. Die Emissionshandelsstelle wurde somit innerhalb des Umweltbundesamtes als zuständige Behörde gemäß der EURichtlinie 2003/87/EG etabliert. Die Aufgaben der Deutschen Emissionshandelsstelle (DEHSt) sind „die Zuteilung und Ausgabe der Emissionsberechtigungen, Aufsicht und Überwachung, die Aufrechterhaltung der nationalen Register sowie nationale und internationale Berichterstattung" (Ziesing 2009: 355).

Nachdem der allgemeine Rahmen des EHS durch das TEHG etabliert worden war, wurden die unterschiedlichen Interessen der Bundesländer innerhalb des politischen Prozesses zur Verabschiedung des Nationalen Allokationsplans (NAP) extrem sichtbar. Der NAP sollte das nationale Emissionsbudget für den Zeitraum 2005-2008 festlegen, was die Entscheidung beinhaltete, die Gesamtmenge der zuzuteilenden Zertifikate (Makro-Plan) und die Regeln für die Menge der Emissionsberechtigungen auf der Grundlage der gewährten verfügbaren Daten (Mikro-Plan) zu definieren (Ziesing 2009: 357). Der NAP der Mitgliedstaaten musste der EU-Kommission zugesandt werden, die den NAP nach definierten Kriterien überprüfte. Hierbei hatte die Kommission die Autorität, die Mitgliedstaaten zur Überarbeitung des NAP zu verpflichten, sollte dieser nicht die EU-Anforderungen erfüllen (Hartmann 2004: 41). Vom EU-Emissionshandelssystem wurden die gesamten Treibhausgasemissionen ebenso wie die $\mathrm{CO}_{2}$-Emissionen erfasst, welche zwischen den Bundesländern extrem variieren (siehe Abb. 7). Daher verhandelten die Bundesländer gemeinsam mit den 
Abb. $7 \mathrm{CO}_{2}$-Emissionen der emissionshandelspflichtigen Anlagen 2009, verteilt auf die Bundesländer. (DEHSt 2010, 63)

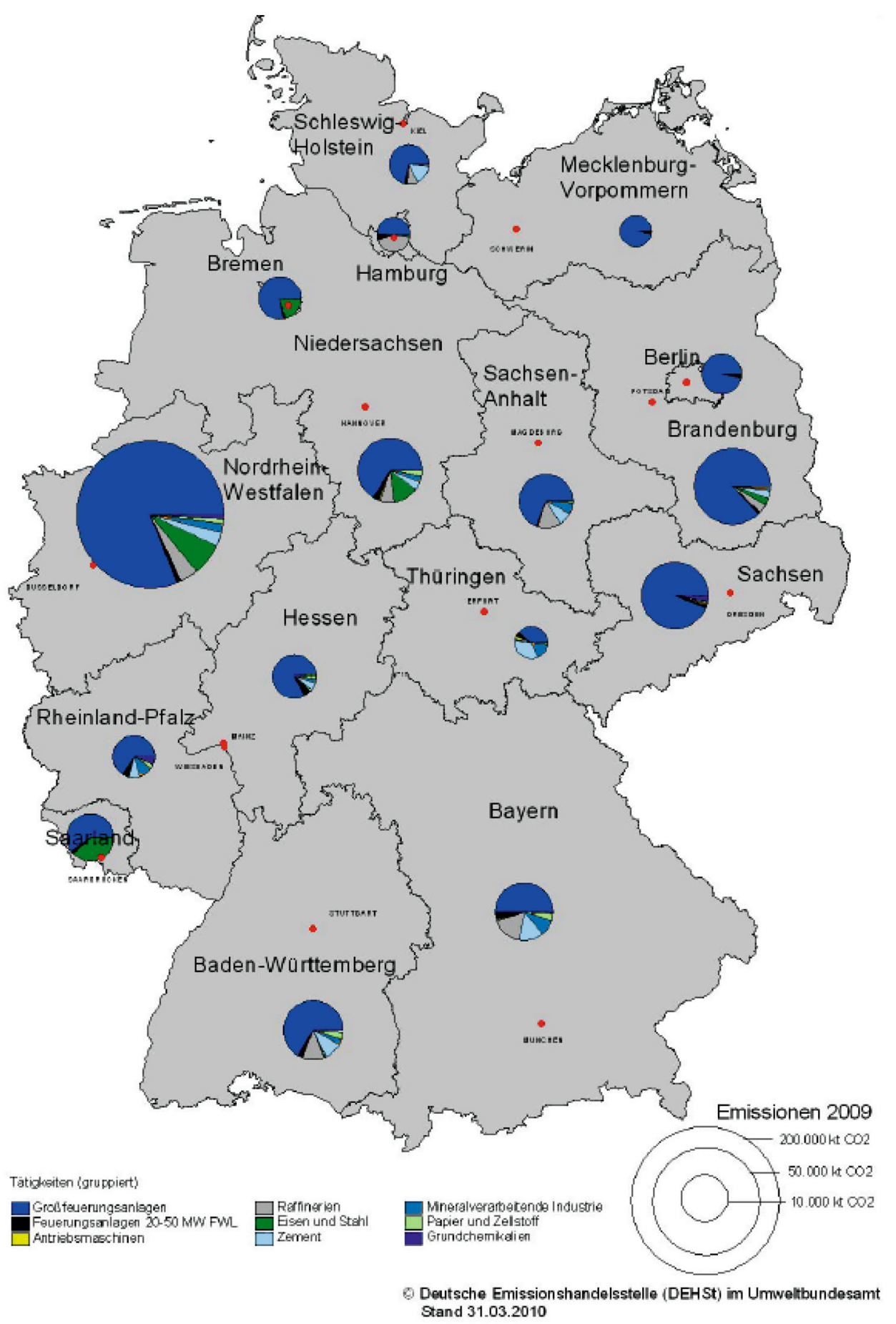

betroffenen Industrien intensiv um die spezifischen EHSVerordnungen und erreichten zugunsten ihrer heimischen wirtschaftlichen Interessen erfolgreich die Anpassung der Vorschriften.

Unter anderem wurden folgende Sonderregelungen zugunsten der Energieindustrie in verschiedenen Bundesländern festgelegt:
- Eine besondere Regel berücksichtigte die vergangenen Reduktionen der Treibhausgasemissionen. Diese Regelung zu 'early actions' war vor allem wichtig für Ostdeutschland, wo umfangreiche Emissionsreduktionen Anfang der 1990er-Jahre durchgeführt worden waren (Schleich/Eichhammer/Böde et al. 2001). Besonders die neuen Bundesländer mit einem hohen Anteil 
an Kohlekraftwerken profitierten von dieser Sonderregel (Michaelowa 2004: 327).

- Die südlichen Bundesländer profitierten von Vorteilen für all jene Kraftwerke, welche die auslaufenden Kernkraftwerke ersetzen sollten. Alle Kraftwerke als Ersatz für Kernkraftwerke würden den Vorteil der kostenlosen Zertifikate erhalten (Michaelowa 2004: 325-327).

- Ebenfalls enthalten war ein brennstoffpezifischer Benchmark $^{11}$ für neue Kraftwerke, der Kohlekraftwerke privilegierte und auf Druck von Nordrhein-Westfalen eingeführt wurde (Ziesing 2009: 364).

Aufgrund der vielfältigen Ausnahmeregelungen des NAP wurde seine Wirksamkeit stark untergraben. Dies geschah im Einklang mit den Interessen vieler Bundesländer, in denen der Wert der Zertifikate nur etwa 1-5\% unter dem Business-as-usual-Szenario für den Industriesektor lag (Michaelowa 2004). In der Folge bewirkte die großzügige Zuteilung nahezu den Zusammenbruch des deutschen EHS.

Der NAP für den nächsten Zeitraum (2008-2012) wurde im Jahr 2006 verabschiedet. Mehrere Änderungen im Vergleich zu dem ersten NAP wurden getätigt, z. B. gab es keine Gewährung von Zulagen auf der Basis von 'early actions' und keine weitere Vergütung für einen Atomausstieg mehr (Kemfert/Diekmann 2006: 666). Aber auch hier wurde die Menge der Emissionsberechtigungen sehr großzügig gewählt. Nur aufgrund der Interventionen der EU-Kommission musste die von der Bundesregierung festgelegte Obergrenze von 482 Mio. t/a auf 453 Mio. t/a reduziert werden (Ziesing 2009: 370).

Für die laufende Periode von 2013-2017 wurde das EHS völlig verändert. Eine EU-weite Obergrenze wurde eingeführt (also nicht eine für jeden Mitgliedstaat), und die $\mathrm{Zu}$ teilung von Zertifikaten basiert auf harmonisierten Regeln. Der wichtigste Mechanismus zur Zuteilung von Zertifikaten ist die Versteigerung (Skjaerseth/Wettestad 2010: 317). Im deutschen Stromsektor erfolgt anstelle einer kostenlosen Zuteilung der Zertifikate eine Versteigerung aller Zertifikate (DEHSt 2010).

Allerdings leidet der Emissionshandel, teilweise auch aufgrund der ökonomischen Krise in Europa, durch eine deutliche Überallokation von Zertifikaten und in der Folge an einem Verfall der Zertifikatspreise. Die ursprünglich anvisierte Anreizsetzung für einen schrittweisen Ausstieg aus der fossilen Energiewirtschaft wurde damit unterlaufen. Anfang des Jahres 2014 stimmte der Europäische Rat daher der Initiative der EU-Kommission zu, Zertifikate aus dem

\footnotetext{
11 Dieser gibt als Leitgröße vor, welche Emissionen pro Produkteinheit (z. B. Gramm $\mathrm{CO}_{2}$ je Kilowattstunde) für die Zuteilung von Emissionsrechten angerechnet werden können. Gelten diese generell und brennstoffübergreifend, führt das dazu, dass für Energieträger mit einem geringeren Ausstoß weniger Emissionszertifikate erworben werden müssen (vgl. SRU 2006: 7 f.).
}

Markt herauszunehmen und erst zu einem späteren Zeitpunkt zur Verfügung zu stellen ('backloading'). Demnach werden 900 Millionen Zertifikate, die zwischen 2014 und 2016 auktioniert werden sollten, zurückgehalten und zum Ende der Handelsperiode 2019-2020 dem System wieder zugeführt.

Bund und Länder lehnten von Beginn an eine Einführung des EHS ab. Nach dem EU-Beschluss 2003 stand die deutsche Politik - auch vor dem Hintergrund der internationalen klimapolitischen Verpflichtungen - aber sehr stark unter Zugzwang. Interventionen von Seiten der Bundesländer konnten sich nur noch auf Einzelfragen konzentrieren. Eine grundsätzliche Blockade erschien den politischen Akteuren nicht mehr möglich, weil der politische Schaden für die Bundesregierung zu groß gewesen wäre. Erleichtert wurde die Umsetzung dadurch, dass die EUVorgaben genügend Spielraum ließen, um in der nationalen Umsetzung föderale Verteilungskonflikte über umfangreiche Zugeständnisse an die ökonomischen Standortinteressen der Bundesländer abzuschwächen. Infolgedessen wurde das Instrument zwar eingeführt und institutionell verankert, ohne aber eine materielle Steuerungswirkung zu entfalten. Gleichzeitig bot die institutionelle Verankerung des Emissionshandels auf EU-Ebene aber mittelfristig das Potenzial effektiver klimapolitischer Steuerungsimpulse, da diese trotz regionaler Verteilungswirkungen und -konflikte im deutschen föderalen Verhandlungssystem nicht länger blockiert werden können. Allerdings haben die Verhandlungen zum Backloading 2013/2014 und auch die Verhandlungen zu weiteren Reformen des Emissionshandels deutlich gemacht, dass auch auf der EU-Ebene starke raumbezogene Verteilungskonflikte existieren. Die Verhandlungen auf EUEbene sind mit ähnlichen Verteilungskonflikten konfrontiert, wie sie die Verhandlungen innerhalb des kooperativen föderalen deutschen Systems kennzeichnen; sie haben eine effektive Lösung bislang blockiert.

Die geringe Anreizwirkung des Emissionshandels hat dazu geführt, dass in Deutschland verstärkt auch über eigene nationale Maßnahmen zur Verringerung des Anteils von Kohlekraftwerken in der Stromerzeugung diskutiert wird. Hintergrund der Diskussion ist, dass die deutschen $\mathrm{CO}_{2}$ Emissionen in 2013 relativ deutlich angestiegen sind, was vor allem auf den Ausbau der Kohleverstromung zurückgeführt wird. Im 2014 verabschiedeten Aktionsprogramm Klimaschutz sieht die Bundesregierung daher vor, dass der Stromsektor eine über die bisherigen Zielsetzungen hinausgehende Reduktion von 22 Mio. $\mathrm{t} \mathrm{CO}_{2}$ erbringen muss (BMUB 2014: 28), die Umsetzung dieser Zielsetzung wurde jedoch auch im Klimaschutzbericht im Jahr 2015 wenig konkretisiert (BMUB 2015). Erste Vorschläge von Seiten des BMWi zielen darauf, Emissionsobergrenzen für den Kraftwerkspark insgesamt zu definieren und die Kraftwerksbetreiber entscheiden zu lassen, wie sie die Minde- 
rungsbeiträge erbringen (vgl. Deutscher Bundestag 2014: 6815). Unter anderem angesichts des unklaren Verhältnisses solcher neuen nationalen Emissionsobergrenzen zum EHS und der bislang fehlenden Ausgestaltung etwaiger Sanktionsmechanismen ist aber noch nicht klar, inwieweit hierdurch effektive Anreize zur Dekarbonisierung der Stromerzeugung gesetzt werden können bzw. inwiefern einzelne Bundesländer hier ihre Standortinteressen geltend machen.

\section{Fazit}

Die föderale Politikgestaltung der deutschen Energiewende ist seit den 1990er-Jahren dadurch geprägt, dass die räumliche Allokation von Treibhausgasen nur implizit und zwar im Rahmen verschiedener Handlungsfelder der nationalen Klimapolitik verhandelt wird. Zwar haben neben der Bundesregierung auch alle Bundesländer eigene Klimaprogramme, die darin formulierten Ziele und Maßnahmen stehen aber nicht in einem kohärenten Verhältnis zur nationalen Klimapolitik. Vielmehr wurde bis heute sowohl bei der Reduktion von Treibhausgasen als auch beim Ausbau der erneuerbaren Energien auf eine explizite Regelung verzichtet, die mit dem europäischen 'burden sharing' vergleichbar wäre. In einer solchen hätte über den Beitrag der einzelnen Bundesländer zum nationalen Emissionsminderungsziel und damit über ihre Beteiligung an den Kosten und Nutzen der Energiewende verhandelt werden müssen. ${ }^{12}$ Aufgrund der Dominanz eines sektoralen Ansatzes und der Konzentration wichtiger klimapolitischer Kompetenzen auf der Bundesebene wurden die regionalwirtschaftlichen Interessen der Bundesländer vor allem in den föderalen Verhandlungssystemen zur Gestaltung der konkreten nationalen Regelungen artikuliert.

Hierbei bestätigt die Analyse beider Handlungsfelder wichtige Befunde aus der Föderalismusforschung: Sie verdeutlicht, dass die föderale Politikgestaltung der Energiewende ökonomische Status-quo-Interessen eher privilegiert und effektive und kosteneffiziente Problemlösungen erschwert. Zwar konnten aufgrund der deutschen Förderpolitik erhebliche Kostendegressionen bei ErneuerbarenEnergien-Technologien realisiert werden, allerdings ist die Belastung der Privathaushalte überproportional hoch, während die industriellen Großverbraucher nur eingeschränkt an den Kosten der erneuerbaren Energien beteiligt wurden. Zugleich führt der Verzicht auf einen räumlich koordinierten Ausbau der erneuerbaren Energien zu einer Verlagerung

\footnotetext{
12 Im Unterschied zu Deutschland verhandelten im Föderalstaat Kanada von 1998-2000 die Provinzen und die Nationalregierung in einem gemeinsamen „National Climate Change Process“ - indes wenig erfolgreich - über sektorale und regionale Klimaschutzziele zur Umsetzung des Kyoto-Protokolls (Macdonald/Monstadt/Kern et al. 2011: $58)$.
}

der Stromproduktion auf die lastfernen Räume. Diese geht zwar mit erheblichem regionalwirtschaftlichem Nutzen für die strukturschwachen ländlichen Räume bzw. den nordostdeutschen Raum einher, jedoch erhöhen sich durch den steigenden Bedarf nach einem kostenintensiven (und raumwirksamen) Ausbau der Übertragungsnetze zur Versorgung der Lastzentren zusätzlich die Stromkosten. Zugleich geht die politische Fokussierung auf die erneuerbaren Energien bislang mit einer Vernachlässigung nachfrageseitiger Klimaschutzmaßnahmen einher, mit denen Treibhausgasemissionen besonders kostengünstig reduziert werden können (z. B. Effizienzsteigerungen im Raumwärme-, Stromerzeugungs- oder PKW-Bereich). Im Emissionshandel konnte durch eine lange Lernperiode mit einer Vielzahl von Ausnahmeregeln als Konzession an heterogene regionale Standortinteressen zwar ein potenziell effektiver und kosteneffizienter Steuerungsmechanismus zur Begrenzung von Treibhausgasemissionen etabliert werden, dieser entfaltet indes durch eine Überallokation von Emissionsrechten auf EU-Ebene derzeit kaum Steuerungswirkung.

Die Handlungsfeldanalysen zeigen, dass vorrangig Strategien der Kostenexternalisierung an die privaten Kleinverbraucher, Paketlösungen und Kompensationsmechanismen angewendet werden und offensichtliche redistributive Effekte möglichst vermieden bzw. zumindest in eine ungewisse Zukunft verschoben wurden. Insbesondere beim Emissionshandel hat zusätzlich der Einfluss der EU-Kommission wesentlich zur Auflösung von Entscheidungsblockaden beigetragen. Im Unterschied zu Befunden der Föderalismusforschung spielt ein ausgeprägter ökonomischer und politischer Wettbewerb zwischen den Bundesländern zumindest im Bereich der erneuerbaren Energien eine wesentliche Rolle, weil mit dem Erneuerbaren-Energien-Gesetz ein interregionaler Wettbewerbsmechanismus etabliert wurde, durch den auch die Bundesländer um die Partizipation an der induzierten Mittelverteilung konkurrieren. So versuchen sie die Standortattraktivität für Investitionen und Arbeitsplätze in erneuerbare Energien durch entsprechende planerische Regelungen, Förderprogramme und -einrichtungen zu erhöhen.

Die Untersuchung zeigt aber auch, dass existierende energiewirtschaftliche Disparitäten im deutschen Bundesstaat nicht reduziert wurden. Die klassischen Energieländer profitieren nur zum Teil vom Ausbau der erneuerbaren Energien und auch die räumliche Konzentration karbonintensiver Industrien und Kraftwerke besteht nach wie vor, was eine große Herausforderung für die Anpassung der regionalen Wirtschaftsstrukturen darstellt. Während Brandenburg und Sachsen-Anhalt frühzeitig auf eine Doppelstrategie fossiler und erneuerbarer Energien gesetzt haben, werden negative räumliche Umverteilungseffekte aktuell insbesondere am Beispiel Nordrhein-Westfalens deutlich. So ist Nordrhein-Westfalen im Saldo der EEG-Zahlungs- 
ströme klar das größte „Geberland“, gleichzeitig schrumpft durch die starke Stromeinspeisung aus Windkraftwerken und Fotovoltaikanlagen der Markt für konventionell erzeugten Strom erheblich und die Wettbewerbsfähigkeit von Steinkohle- und Gaskraftwerken sinkt. Insbesondere die aktuellen Gewinneinbrüche und die daraus resultierenden Umstrukturierungen der auf fossile Energieträger fokussierten Energiewirtschaft des Ruhrgebiets machen dies deutlich. Zwar gehen bislang vom Emissionshandel kaum Anreize zur Dekarbonisierung der Energiewirtschaft und damit nur geringe Umverteilungseffekte aus, mittel- bis langfristig ist jedoch auch hier mit Nachteilen für Standorte fossiler Energiebranchen und energieintensiver Industrien zu rechnen.

Die künftige Energiewendepolitik steht vor verschiedenen Herausforderungen: Erstens wird es stärker als bisher darauf ankommen, die Energieversorgung in einem europäischen Mehrebenenkontext zu konzipieren. Durch einen wachsenden regulativen Einfluss der EU ist zu erwarten, dass die Umverteilungskonflikte zwischen den Bundesländern und die hierdurch provozierten föderalen Handlungsblockaden teilweise neutralisiert werden. Angesichts der Verteilungskonflikte zwischen den EU-Mitgliedsländern sind allerdings Hoffnungen auf eine generell höhere klimapolitische Problemlösungsfähigkeit zu relativieren. Angesichts der gegenwärtigen Unwirksamkeit des $\mathrm{CO}_{2}-$ Emissionshandelssystems (EHS) erscheinen die nationalen Klimaziele nur dann realisierbar, wenn die Umweltkosten etwa der Kohleverstromung durch ergänzende nationale Preis- und Marktregulierungen sichtbar gemacht werden. Zweitens stellt sich beim Ausbau der erneuerbaren Energien zunehmend die Frage nach einem in volkswirtschaftlicher und räumlicher Hinsicht optimalen Ausbau. Nötig sind hierbei Entscheidungen, ob sich der Aufbau von regenerativen Stromerzeugungskapazitäten auch künftig vor allem auf ertragreiche bzw. dünn besiedelte, ländliche Standorte konzentrieren und damit ein weiterer kostenintensiver und raumunverträglicher Ausbau der Übertragungsnetze in Kauf genommen werden soll. Alternativ müssten die Stromerzeugung und -nachfrage räumlich besser koordiniert werden (vgl. Agora Energiewende 2013). So müsste die weitere Verlagerung der Stromerzeugung in die „Lastperipherien“" verringert und der Ausbau klimaverträglicher Erzeugungskapazitäten in der Nähe der industriellen Lastzentren verstärkt werden. Die Herausforderung besteht drittens darin, die Anstrengungen zur Ausschöpfung der raumspezifischen Effizienzpotenziale zu erhöhen - einem bislang zu wenig beachteten Bestandteil einer wirklichen Energiewende. Viertens wird über Anreizmechanismen nachzudenken sein, die einen Innovationswettbewerb zwischen den Bundesländern nicht nur beim Ausbau der erneuerbaren Energien, sondern auch hinsichtlich einer verbesserten Koordination zwischen Angebot und Nachfra- ge fördern. Die Fragen, wie ein „intelligenter“ Ausgleich zwischen Nachfrage und Erzeugung auch in räumlicher Hinsicht gewährleistet und wie mit den immer offensichtlicheren regionalen Umverteilungseffekten der Energiewende angesichts der skizzierten Koordinationsprobleme zwischen Bund und Ländern umgegangen werden kann, können an dieser Stelle nicht abschließend beantwortet werden. Institutionelle Anpassungen des föderalen Systems durch effizientere Koordinationsgremien zwischen Bund und Ländern (z.B. Länderarbeitsgemeinschaften, Verstetigung „föderaler Energiegipfel“) sowie eine bessere räumliche Koordination der länderspezifischen Politiken stellen für den Erfolg der Energiewende jedoch zentrale Herausforderungen dar.

Danksagung Die Autoren danken den Partnern eines international vergleichenden Forschungsprojekts zur Allokation von Treibhausgasen in föderalen Systemen, insbesondere Douglas MacDonald und Kristine Kern, sowie den Mitgliedern des ARL-Arbeitskreises „Räumliche Politik und Planung der Energiewende“ für fruchtbare Diskussionen und ihre konstruktiven Kommentare zu früheren Versionen des vorliegenden Artikels. Gedankt sei auch zwei anonymen Gutachtern und Gregor Prinzensing für ihre fachlich fundierten und detaillierten Kommentare.

\section{Literatur}

AGEE-Stat - Arbeitsgruppe Erneuerbare Energien-Statistik (2015): Zeitreihen zur Entwicklung der erneuerbaren Energien in Deutschland. Berlin.

Agora Energiewende (2013): Kostenoptimaler Ausbau der Erneuerbaren Energien in Deutschland. Ein Vergleich möglicher Strategien für den Ausbau von Wind- und Solarenergie in Deutschland bis 2033. Berlin.

Agora Energiewende (2015): Die Energiewende im Stromsektor: Stand der Dinge 2014. Berlin.

Bailey, I.; Maresh, S. (2008): Facing up to the greenhouse challenge? Australian climate politics. In: Compston, H.; Bailey, I. (Hrsg.): Turning down the heat. The politics of climate policy in affluent democracies. London, 202-222.

BBSR - Bundesinstitut für Bau-, Stadt- und Raumforschung (2012): Raumordnungsbericht 2011. Hannover.

BDEW - Bundesverband der Energie- und Wasserwirtschaft e.V. (2014): Erneuerbare Energien und das EEG: Zahlen, Fakten, Grafiken. Berlin.

Benz, A. (1998): Politikverflechtung ohne Politikverflechtungsfalle Koordination und Strukturdynamik im europäischen Mehrebenensystem. In: Politische Vierteljahresschrift 39, 3, 558-589.

Benz, A. (2007a): Inter-regional competition in co-operative federalism: New modes of multi-level governance in Germany. In: Regional and Federal Studies 17, 4, 421-436.

Benz, A. (2007b): Multilevel Governance. In: Benz, A.; Lütz, S.; Schimanek, U.; Simonis, G. (Hrsg.): Handbuch Governance. Wiesbaden, 297-310.

Biedermann, A. (2011): Klimaschutzziele in den deutschen Bundesländern. Dessau-Roßlau. $=$ Climate Change, 15.

Blancke, S. (2004): Politikinnovationen im Schatten des Bundes: Policy-Innovationen und -Diffusionen im Föderalismus und die Arbeitsmarktpolitik der Bundesländer. Wiesbaden.

BMU - Bundesministerium für Umwelt, Naturschutz und Reaktorsicherheit (2013): Zeitreihen zur Entwicklung der Kosten des EEG. Unter Verwendung von durch die Übertragungsnetzbetreiber (ÜNB) veröffentlichten Daten. Berlin. 
BMUB - Bundesministerium für Umwelt, Naturschutz, Bau und Reaktorsicherheit (2014): Aktionsprogramm Klimaschutz 2020. Eckpunkte des BMUB. Berlin.

BMUB - Bundesministerium für Umwelt, Naturschutz, Bau und Reaktorsicherheit (2015): Klimaschutzbericht 2015. Zum Aktionsprogramm Klimaschutz 2020 der Bundesregierung. Berlin.

BMWI - Bundesministerium für Wirtschaft und Energie (2015): Hintergrundinformationen zur Besonderen Ausgleichsregelung. Antragsverfahren 2014 auf Begrenzung der EEG-Umlage 2015. Berlin.

Braun, D. (2004): Föderalismus. In: Helms, L.; Jun, U. (Hrsg.): Politische Theorie und Regierungslehre. Frankfurt am Main, New York, 130-162.

Breyer, C.; Müller, B. (2013): Vergleich und Optimierung von zentral und dezentral orientierten Ausbaupfaden zu einer Stromversorgung aus erneuerbaren Energien in Deutschland. Berlin.

Bruns, E.; Ohlhorst, D.; Wenzel, B.; Köppel, J. (2011): Renewable Energies in Germany's Electricity Market. A Biography of the Innovation Process. Dordrecht u. a.

Büsgen, U.; Dürrschmidt, W. (2009): The expansion of electricity generation from renewable energies in Germany. In: Energy Policy 37, 7, 2536-2545.

Bulkeley, H.; Broto, V. C.; Edwards, G. (2012): Bringing climate change to the city: towards low carbon urbanism? In: Local Environment 17, 5, 545-551.

Bundesrat (2004): Stenografischer Bericht zur 798. Sitzung des Bundesrates vom 2. April 2004. Berlin.

Crowley, K. (2007): Is Australia faking It? The Kyoto protocol and the greenhouse policy challenge. In: Global Environmental Politics 7, 4, 118-139.

DEHSt - Deutsche Emissionshandelsstelle (2010): Kohlendioxid emissionen der emissionshandelspflichtigen stationären Anlagen und im Luftverkehr in Deutschland im Jahr 2010. Berlin.

Deutscher Bundestag (2014): Stenografischer Bericht der 72. Sitzung des Deutschen Bundestags (BT-PIPr. 18/72). Tagesordnungspunkt 2: Befragung der Bundesregierung: Erster Fortschrittsbericht - Energiewende und Nationaler Aktionsplan Energieeffizienz. Berlin, 6812-6825.

Diller, C. (2015): Koordination in der Regionalplanung: Theoretische Überlegungen, empirische Ergebnisse und Forschungsperspektiven. In: Karl, H. (Hrsg.): Koordination raumwirksamer Politik. Mehr Effizienz und Wirksamkeit von Politik durch abgestimmte Arbeitsteilung. Hannover, 113-130. = Forschungsberichte der ARL, 4.

Ehlers, A.; Böhme, R. (2011): Windenergie in der Landesplanung. In: Natur und Recht 33, 5, 323-329.

Gawel, E.; Korte, K. (2015): Regionale Verteilungswirkungen und Finanzierungsverantwortung: Bund und Länder bei der Strom-Energiewende. In: Müller, T.; Kahl, H. (Hrsg.): Energiewende im Föderalismus. Baden-Baden, 145-186.

Haas, M.; Jun, U.; Niedermayer, O. (Hrsg.) (2008): Parteien und Parteiensysteme in den deutschen Ländern. Wiesbaden.

Hanf, K.; Morata, F. (2012): Spanish subnational involvement in multi-level sustainable development issues. In: Bruyninckx, H.; Happaerts, S.; van den Brande, K. (Hrsg.): Sustainable development and subnational governments. Policy-Making and Multi-Level Interactions. London, 141-159.

Harrison, K. (2007): The road not taken: Climate change policy in Canada and the United States. In: Global Environmental Politics 7, $4,92-117$.

Hartmann, D. (2004): Die Umsetzung der EU-Emissionshandelsrichtlinie in der Bundesrepublik Deutschland. Berlin (Diplomarbeit, Freie Universität Berlin).

Hegele, Y.; Behnke, N. (2013): Die Landesministerkonferenzen und der Bund - Kooperativer Föderalismus im Schatten der Politikverflechtung. In: Politische Vierteljahresschrift 54, 1, 21-49.
Hellmann, T.; Kullas, M. (2012): Das ökonomische Innovationsregime der Bundesländer im föderalen Deutschland. In: Härtel, I. (Hrsg.): Handbuch Föderalismus. Band II. Heidelberg, 137-164.

Hirschl, B. (2008): Erneuerbare Energien-Politik. Eine Multi-Level Policy-Analyse mit Fokus auf den deutschen Strommarkt. Wiesbaden.

Hrbek, R. (2004): Germany. In: Griffiths, A. L. (Hrsg.): Handbook of federal countries. Montréal, 150-164.

Jacobsson, S.; Lauber, V. (2006): The Politics and policy of energy system transformation - Explaining the German diffusion of renewable energy technology. In: Energy Policy 34, 3, 256-276.

Jakubowski, P.; Zarth, M. (2009): Regionale Produktionsverlagerungen durch den $\mathrm{CO}_{2}$-Emissionshandel. Bonn. = BBSR-Berichte Kompakt, 7/2009.

Jörgensen, K. (2012): Governance for sustainable development in the German Bundesländer. In: Bruyninckx, H.; Happaerts, S.; van den Brande, K. (Hrsg.): Sustainable Development and Subnational Governments. Policy-Making and Multi-Level Interactions. London, 103-119.

Kemfert, C.; Diekmann, J. (2006): Europäischer Emissionshandel Auf dem Weg zu einem effizienten Klimaschutzinstrument. In: Wochenbericht des DIW Berlin 73, 661-675.

Kern, K. (2008): Sub-national sustainable development initiatives in federal states in Germany. In: Baker, S.; Eckerberg, K. (Hrsg.): In pursuit of sustainable development. London, 122-144.

Kropp, S. (2010): Kooperativer Föderalismus und Politikverflechtung. Wiesbaden.

LAK Energiebilanzen - Länderarbeitskreis Energiebilanzen (2016): Ergebnisse und Zeitreihen. http://www.lak-energiebilanzen.de (11.3.2016)

Landesregierung Saarland (1998): Klimagutachten. Saarbrücken.

Lehmbruch, G. (2000): Parteienwettbewerb im Bundesstaat. Wiesbaden.

Macdonald, D. (2008): Explaining the failure of Canadian climate policy. In: Compston, H.; Bailey, I. (Hrsg.): Turning down the heat. The politics of climate policy in affluent democracies. London, 223-240.

Macdonald, D.; Monstadt, J.; Kern, K.; Hayden, A.; Gordon, D.; Bidordinova, A.; Scheiner, S.; Pristupa, A. (2011): Allocating Canadian greenhouse gas emission reductions amongst sources and provinces: Learning from Germany and the EU. Toronto.

Meuser, M.; Nagel, U. (2005): ExpertInneninterviews. Vielfach erprobt, wenig bedacht. In: Bogner, A.; Littig, B.; Menz, W. (Hrsg.): Das Experteninterview. Theorie, Methode, Anwendung. Wiesbaden, 71-93.

Mez, L. (2003): Ökologische Modernisierung und Vorreiterrolle in der Energie- und Umweltpolitik? Eine vorläufige Bilanz. In: Egle, C.; Ostheim, T.; Zohlnhöfer, R. (Hrsg.): Das rot-grüne Projekt. Wiesbaden, 329-350.

Michaelowa, A. (2004): Großzügige Versorgung der Großemittenten mit $\mathrm{CO}_{2}$-Emissionsrechten. In: HWWA-Wirtschaftsdienst 84, 5, 325-328.

Michaelowa, A. (2005): The German wind energy lobby: How to promote costly technological change successfully. In: European Environment $15,192-199$.

Monstadt, J. (2004): Die Modernisierung der Stromversorgung. Regionale Energie- und Klimapolitik im Liberalisierungs- und Privatisierungsprozess. Wiesbaden.

Monstadt, J. (2007): Energiepolitik und Territorialität. Internationalisierung und Regionalisierung der Energieversorgung als Herausforderung staatlicher Steuerung. In: Gust, D. (Hrsg.): Wandel der Stromversorgung und räumliche Politik. Hannover, 186-216. = Forschungs- und Sitzungsberichte der Akademie für Raumforschung und Landesplanung, 227.

Monstadt, J.; Scheiner, S. (2014): Allocating greenhouse gas emissions in the German federal system: Regional interests and federal climate governance. In: Energy Policy 74, 11, 383-394. 
O’Sullivan, M.; Edler, D.; Bickel, P.; Lehr, U.; Peter, F.; Sakowski, F. (2014): Bruttobeschäftigung durch erneuerbare Energien in Deutschland im Jahr 2013 - eine erste Abschätzung. o. O.

Peterson, T.; Rose, A. (2006): Reducing conflicts between climate policy and energy policy in the US: The important role of the states. In: Energy Policy 34, 5, 619-631.

Prognos (2011): Bedeutung der Braunkohle in Ostdeutschland. Berlin.

Rabe, B. G. (2009): Second-generation climate policies in the states: Proliferation, diffusion, and regionalization. In: Selin, H.; VanDeveer, S. D. (Hrsg.): Changing climates in North American politics. Institutions, policymaking, and multilevel governance. Cambridge, 67-85.

Rodi, M.; Sina, S. (2011): Das Klimaschutzrecht des Bundes - Analyse und Vorschläge zu seiner Weiterentwicklung. Dessau-Roßlau. = Climate Change, 17/2011.

Scharpf, F. W. (1976): Theorie der Politikverflechtung. In: Scharpf, F.; Reissert, W.; Schnabel, B. (Hrsg.): Politikverflechtung. Theorie und Empirie des kooperativen Föderalismus in der Bundesrepublik. Kronberg/Ts., 13-70.

Scharpf, F. W. (2009): Föderalismusreform: Kein Ausweg aus der Politikverflechtungsfalle? Frankfurt am Main.

Scheller, H. (2008): Ursprünge und Rezeption des Politikverflechtungsansatzes - Auswanderung aus der Wissenschaft und politische Instrumentalisierung. In: Scheller, H.; Schmidt, J. (Hrsg.): Föderale Politikgestaltung im deutschen Bundesstaat - Variable Verflechtungsmuster in Politikfeldern. Baden-Baden, 13-35.

Schlager, E.; Engel, K.; Rider, S. (Hrsg.) (2011): Navigating climate change policy. The opportunities of federalism. Tucson.

Schleich, J.; Eichhammer, W.; Böde, U.; Gagelmann, F.; Jochem, E.; Schlomann, B.; Ziesing, H.-J. (2001): Greenhouse gas reductions in Germany - lucky strike or hard work? In: Climate Policy 1, 3, 363-380.

Skjaerseth, J.; Wettestad, J. (2008): EU emissions trading. Initiation, decision-making and implementation. Aldershot.

Skjaerseth, J.; Wettestad, J. (2010): Making the EU emissions trading system: The European Commission as an entrepreneurial epistemic leader. In: Global Environmental Change 20, 2, 314-321.

Smart Grids Plattform Baden-Württemberg (2013): Roadmap der Smart Grids-Plattform Baden-Württemberg. Stuttgart.
SRU - Sachverständigenrat für Umweltfragen (2006): Die nationale Umsetzung des europäischen Emissionshandels: Marktwirtschaftlicher Klimaschutz oder Fortsetzung der energiepolitischen Subventionspolitik mit anderen Mitteln? Berlin.

Statistik der Kohlenwirtschaft e. V. (2015): Der Kohlenbergbau in der Energiewirtschaft der Bundesrepublik Deutschland im Jahre 2014. Herne und Köln.

Suck, A. (2005): The politics for a sustainable energy industry: Renewable energy policy in the United Kingdom and in Germany. In: Coen, D.; Héritier, A. (Hrsg.): Refining regulatory regimes. Utilities in Europe. Cheltenham, 147-182.

Suck, A. (2008): Erneuerbare Energien und Wettbewerb in der Elektrizitätswirtschaft: staatliche Regulierung im Vergleich zwischen Deutschland und Großbritannien. Wiesbaden.

Tàbara, J. D. (2007): A new climate for Spain: Accommodating environmental foreign policy in a federal state. In: Harris, P. G. (Hrsg.): Europe and global climate change. Politics, Foreign Policy and Regional Cooperation. Cheltenham, 161-180.

Ulrich, P.; Lehr, U. (2014): Erneuerbar beschäftigt in den Bundesländern: Bericht zur aktualisierten Abschätzung der Bruttobeschäftigung 2013 in den Bundesländern. Osnabrück.

Wachendorfer-Schmidt, U. (2005): Politikverflechtung im vereinigten Deutschland. Wiesbaden.

Weidner, H.; Eberlein, B. (2009): Still walking the talk? German climate change policy and performance. In: Eberlein, B.; Doern, G. B. (Hrsg.): Governing the energy challenge. Canada and Germany in a multilevel regional and global context. Toronto, 314-343.

Wurzel, R. (2010): Environmental, climate and energy Policies: Pathdependent incrementalism or quantum leap? In: German Politics 19, 3/4, 460-478.

Ziesing, H.-J. (2009): EU emission trading and national allocations plans 2005-2007: The case of Germany. In: Eberlein, B.; Doern, G. B. (Hrsg.): Governing the energy challenge. Canada and Germany in a multilevel regional and global context. Toronto, 344-372.

Zimmer, C. (2010): Politikkoordination im deutschen Bundesstaat: Wandel in den Arbeitsstrukturen? In: Zeitschrift für Parlamentsfragen 41, 3, 677-692.

Zimmermann, H. (2005): Regionale Effekte einer $\mathrm{CO}_{2}$-Zertifikate-Politik - ein Problemaufriss. In: Raumforschung und Raumordnung 63, 4, 279-287. 